Check for updates

Cite this: Mater. Chem. Front. 2020, 4, 2881

Received 31st May 2020

Accepted 10th July 2020

DOI: 10.1039/d0qm00365d

rsc.li/frontiers-materials

\title{
Structure-property relationship of assembled nanowire materials
}

\author{
Rui Wang, Cheng Chen, Yi Zheng, Heng Wang, Jian-Wei Liu (D) and \\ Shu-Hong Yu (D)*
}

\begin{abstract}
Due to their unique structure, one dimensional (1D) nanomaterials have attracted considerable attention and shown potential applications in modern nano-devices. With advances in chemistry and materials science, integrating these 1D nanoscale-building blocks for macroscopic well-designed assemblies will provide new opportunities for sustainable and advanced applications. This article comprehensively reviews the structure-property relationship of assembled nanowire materials compared with the disordered NW statement. Specifically, we firstly highlight the anisotropy phenomenon of 1D assemblies, including the anisotropic conductive properties, optical properties, thermal conduction properties, and anisotropic strain sensing. Next, we systematically review the superior properties of ordered 1D assembly devices compared to random samples to elucidate the advantages of well-defined structures. The programmability of ordered assemblies with predictable architectures for large-scale devices is also briefly commented on. Finally, a summary and perspectives on challenges and future opportunities are presented.
\end{abstract}

\section{Introduction}

One-dimensional (1D) nanomaterials, such as nanowires (NWs), nanotubes (NTs), and nanorods (NRs), show great potential as one of the most promising material platforms for next-generation nano-devices, such as optical devices, ${ }^{1}$ electronics, ${ }^{2}$ sensors, ${ }^{3}$ energy harvesting, ${ }^{4}$ and catalysis, ${ }^{5}$ owing to their unique electronic and optical properties. ${ }^{6-8}$ The structural characteristics (i.e., large aspect ratio) are beneficial to optimize the propagating pathways for quantum particles (electrons, phonons, and photons). On the basis of the chemical compositions and electronic properties, NWs can be categorized into metallic, semiconducting, and dielectric NWs, where metallic and dielectric nanowires are suitable for plasmonic and photonic waveguiding applications and semiconducting NWs are promising for light generation and sensing applications. ${ }^{9-12}$ At the beginning of nanowire research, scientists firstly focused on the synthesis of 1D nanomaterials. At the 1990s, several groups initiated research using a solution-liquid-solid process, vaporliquid-solid strategy, and vapor phase conversion and transport process. ${ }^{13-18}$ Meanwhile, some research interest gradually shifted from controlled synthesis to integration of these NWs

Division of Nanomaterials \& Chemistry, Hefei National Laboratory for Physical Sciences at Microscale, Institute of Energy, Hefei Comprehensive National Science Centre, Hefei Science Centre, CAS Center for Excellence in Nanoscience, Department of Chemistry, Institute of Biomimetic Materials \& Chemistry, University of Science and Technology of China, Hefei, 230026, China. E-mail: jwliu13@ustc.edu.cn, shyu@ustc.edu.cn for complicated structures which can not only retain the single unit properties but induce new macroscopic properties such as electric conduction, optical properties, and thermal conductivity. ${ }^{19-23}$ During this period, different assembly strategies were developed including spray coating, ${ }^{24}$ blade coating, ${ }^{25}$ Langmuir-Blodgett (LB), ${ }^{26,27}$ drop-casting, ${ }^{28,29}$ solution shearing, ${ }^{30}$ and external electric/magnetic fields ${ }^{31,32}$ to arrange 1D nanomaterials with specific architecture to obtain functionality. Benefiting from this, metal NWs were assembled for transparent conducting electrodes (TCEs) with high flexibility to replace rigid indium tin oxide. ${ }^{33,34}$ Carbon nanotubes (CNTs) were aligned for advanced nano-processors for higher processing speed. ${ }^{35,36}$ Compared to the random configuration of $1 \mathrm{D}$ nanomaterials, aligning them with ordered structure and uniform orientation can not only introduce a dramatic increase in surface area/volume ratio but also cause emergence of collective properties as a result of the arrangement or assembly. ${ }^{37,38}$ As presented in Fig. 1, firstly, 1D nanomaterials with the same orientation can have anisotropic features due to the intrinsic $2 \mathrm{D}$ confinement structures, which endow one material dualproperties along the orthogonal direction. Secondly, the ordered assembly could change the microstructure of the 1D nanomaterial network to obtain a superior device performance compared to the random configuration. Furthermore, with precise assembly technology, the types and location of $1 \mathrm{D}$ nanomaterials can be controlled for sophisticated nano-micro structure, which is crucial for the practical application of 1D nanomaterial-based high-precision devices, such as artificial 


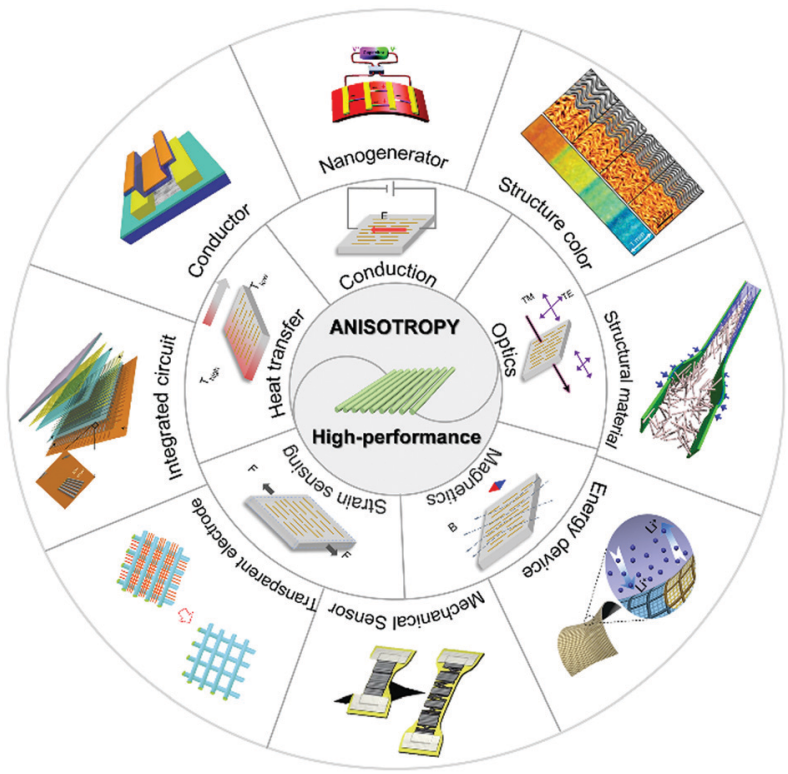

Fig. 1 Superiority of the ordered 1D nanomaterial assembly. Reprinted with permission from ref. 63 and 125. Copyright 2018 and 2014 Wiley. Reprinted with permission from ref. 119 and 131. Copyright 2010 and 2011 Nature Publishing Group. Reprinted with permission from ref. 138 and 168. Copyright 2010 and 2017 American Chemical Society. Reprinted with permission from ref. 144 and 148. Copyright 2011 and 2014 Nature Publishing Group.

skin, integrated photosensors and so on. ${ }^{39,40}$ This designable 1D nanomaterial structure can also regulate macroscopic assemblies to obtain alterable characteristics, such as NW structural color (SC) of NWs. The merits of assembled NW structures are revealed in Fig. 2.

Recently, several excellent review papers have reported the development of 1D nanomaterial assembly, which placed more focus on the assembly methods without covering the effect induced by ordered assembly. ${ }^{39,41-52}$ To address this issue, we provide a comprehensive summary of the typical anisotropic effects induced by the alignment of 1D nanomaterials. The performance improvement of several 1D nanomaterial devices with ordered configuration is also overviewed, such as flexible conductors, TCEs, strain sensors, and nanogenerators. Overall, the advantages of ordered assembly are discussed in detail in this review. We anticipate that this review is useful for research focused on the ordered assembly of 1D nanomaterials.

\section{Anisotropy of ordered 1D nanomaterial-based assemblies}

1D nanomaterials show anisotropic properties with a 1D wirestructure. $^{53-55}$ To extend these properties to macroscopic assemblies, controllable assembly is essential. With all the nanomaterials aligned with the same orientation, intrinsic anisotropy of 1D nanomaterials will be retained. We will present in this section the typical macroscopic anisotropy derived from ordered 1D nanomaterial assembly.

\subsection{Anisotropy of conductive properties}

Anisotropic conductive materials using 1D nanomaterials show different conductivity in a different direction. ${ }^{56-58}$ In the NW network, the conductivity is affected by two factors, i.e., the inherent material resistance and the junction resistance between NWs. With a disordered configuration, carriers can be transported along all directions, showing isotropic conductivity. In an assembly network, the resistance of the NW array is dominated by the gap of two adjacent NWs. In a typical aligned NW array, all the NWs show head to tail alignment along the axial direction with the least junctions. Along the NWs show side-by-side morphology. Because of the anisotropic shape of

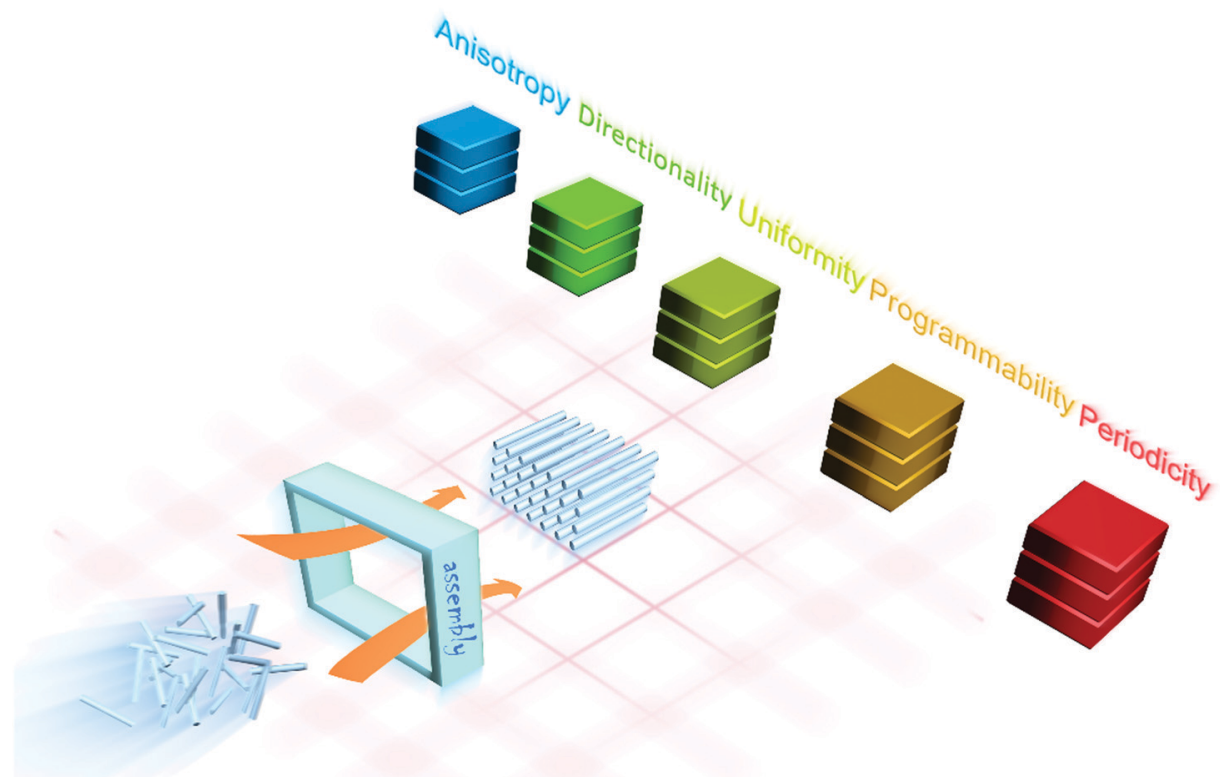

Fig. 2 Schematic illustration of the merits of ordered 1D nanomaterials assembly. 
a)

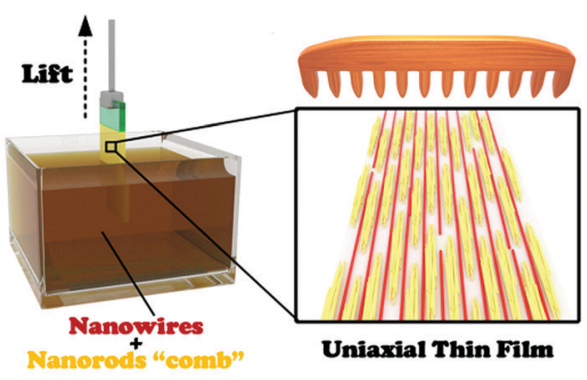

d)

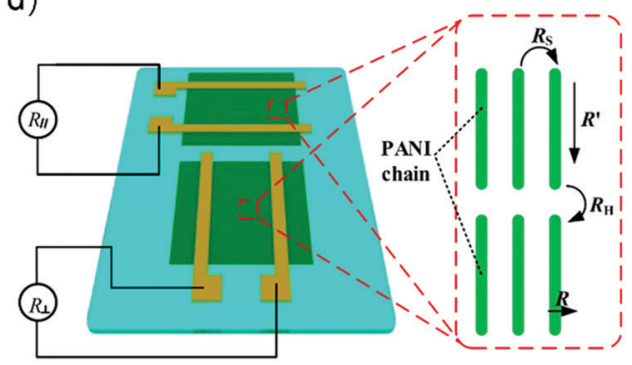

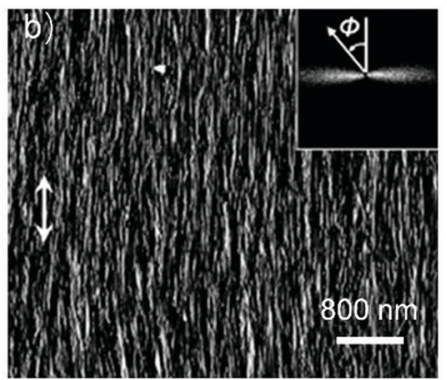

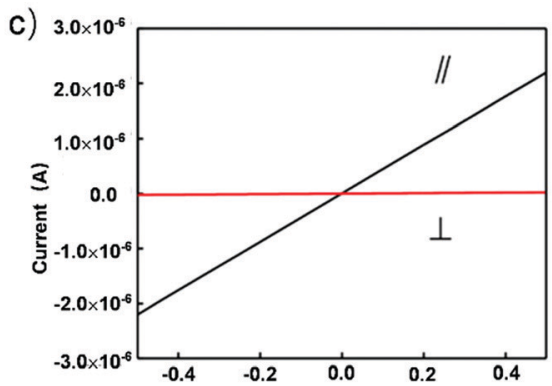

e)

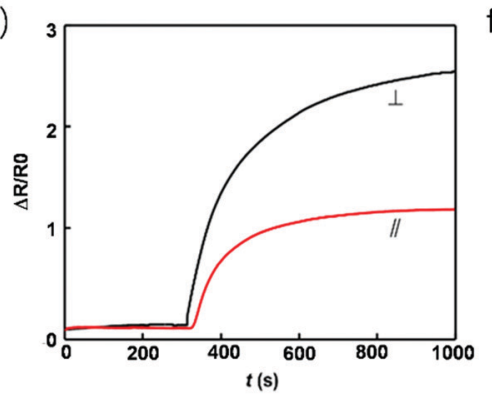

f)

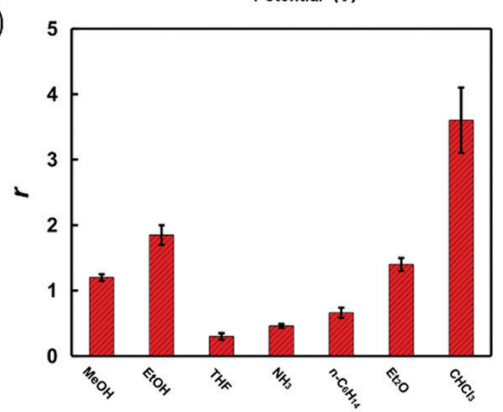

g)

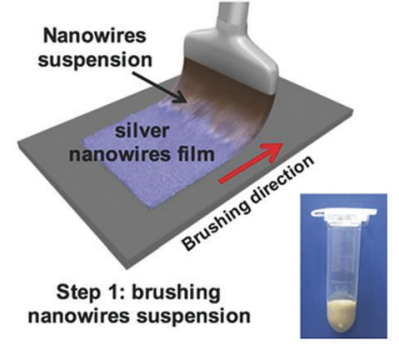

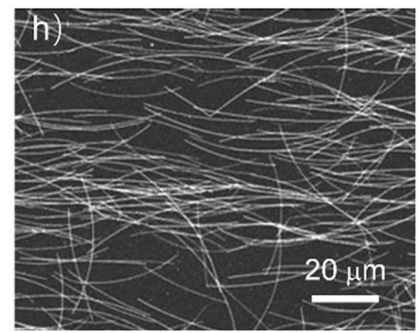
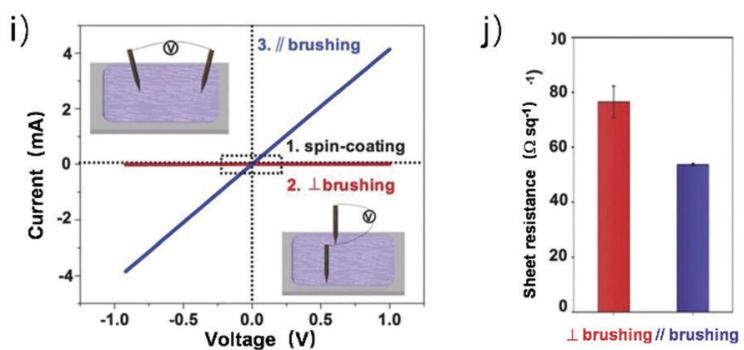

Fig. 3 Anisotropy of the electrical conductivity in ordered NW structures. (a) Preparation of the $\mathrm{V}_{2} \mathrm{O}_{5} \cdot n \mathrm{H}_{2} \mathrm{O}-\mathrm{CNC}$ thin film by the dip-coating method. (b) AFM image of the aligned $\mathrm{V}_{2} \mathrm{O}_{5} \cdot n \mathrm{H}_{2} \mathrm{O}-\mathrm{CNC}$ thin film (insert, FFT image). (c) Current-potential curves for the PANI-CNC thin film in the parallel ( $\|$ ) and vertical $(\perp)$ directions relative to the shearing direction. (d) Schematic diagram of the gas sensor. (e) The degrees of resistance variation of the gas sensor when testing $\mathrm{NH}_{3}$. (f) The ratio of resistance changes $\left(\Delta R / R_{0}\right)$ between the two directions for different gases. (g) Preparation of the Ag NW film by CFAbrush coating. (h) SEM image of ordered Ag NWs. (i) Current-voltage curves for the CFA-brushed film and spin-coated film. (j) The sheet resistance of the CFA-brushed film along two directions. (a-f) Reprinted with permission from ref. 59. Copyright 2018 American Chemical Society. (g-j) Reprinted with permission from ref. 30. Copyright 2018 Wiley.

the NWs, more NWs along the radical direction could be counted in the defined area than the axial direction, which also means that the junctions and gaps are more in the radical direction and induces larger resistance along this orientation. The anisotropic conductivity brings a dual electrical property to the new materials, which could be applied for multidimensional sensors.

Nanoscale ordered nano-fibrillar polyaniline (PANI) was fabricated by Bai and co-workers using the dip-coating method. ${ }^{59}$ As shown in Fig. 3a, a simple assembly process was performed by immersing a vertically oriented substrate in a $\mathrm{V}_{2} \mathrm{O}_{5} \cdot n \mathrm{H}_{2} \mathrm{O}$-cellulose nanocrystal $(\mathrm{CNC})$ solution and then lifting it. The thin film with aligned nano-fibrillar PANI is presented in Fig. 3b. Because of the orientational structure, the resistance of PANI in the perpendicular direction was significantly higher than that in the direction parallel to the shearing direction (Fig. 3c). Moreover, the anisotropic performance of films could be used in a gas sensor (Fig. 3d). Once the device was exposed to various atmospheres, $R_{\|}$and $R_{\perp}$ started to change concurrently. However, the degree of resistance variation was different due to the different response
(Fig. 3e). The ratios of change $\left(\Delta R / R_{0}\right)$ in the two orthogonal directions had specificity towards a specific gas. Utilizing this property of the assembly device, the authors constructed a highly selective gas sensor to distinguish multiple gases without a concentration effect through ratios of relative resistance change (Fig. 3f).

An electrically anisotropic film can also be fabricated by brush painting. Highly transparent flexible electrodes (TFEs) containing ordered Ag NW arrays by utilizing directional liquid transfer were demonstrated by Liu et al. ${ }^{30}$ As shown in Fig. 3g, $\mathrm{Ag}$ NW solution was brushed onto a poly(ethylene terephthalate) (PET) substrate by a conical fiber array (CFA) to form an aligned NW network. The PET substrate with ordered Ag NW coating is shown in Fig. $3 \mathrm{~h}$. The significant differences in electrical conductivity between the CFA-brush coating and the spin coating are shown in Fig. $3 \mathrm{i}$ and j. Besides, the conductivity of the CFA-brushed TFEs was greatly improved in the parallel direction, which was 270 times higher than the isotropic Ag NW electrodes produced by spin coating. Moreover, 
a)

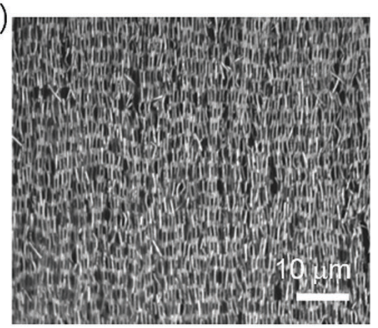

b)

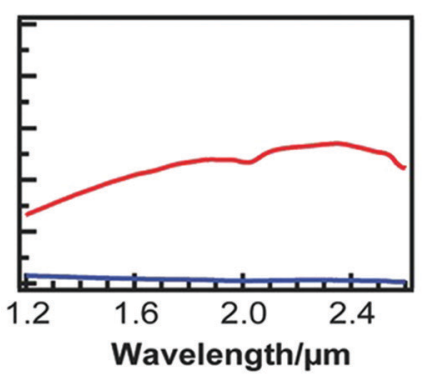

c)

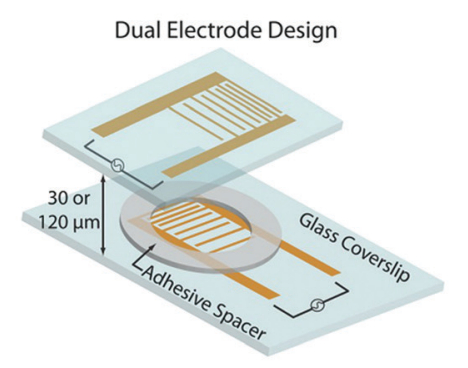

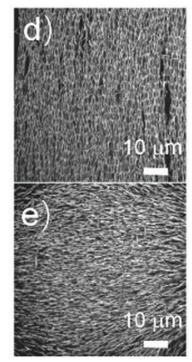

f)

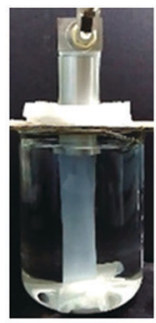

j)

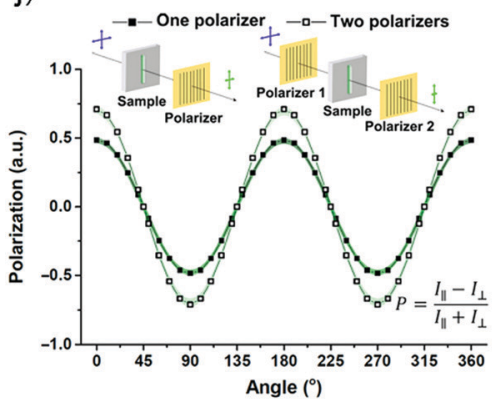

g)

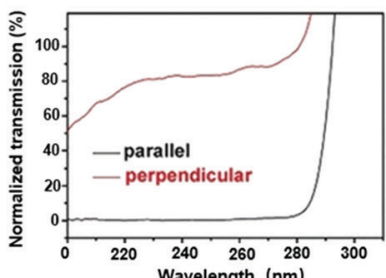

k)

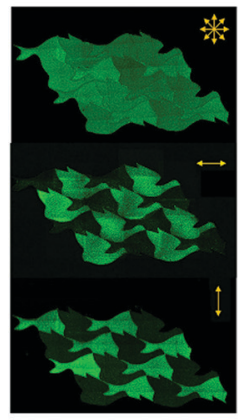

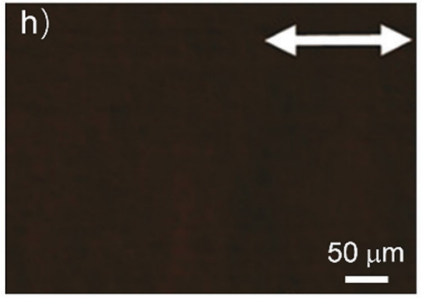

I)

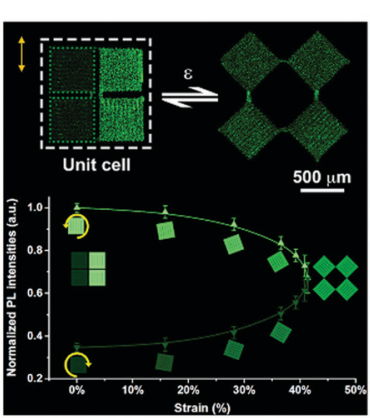

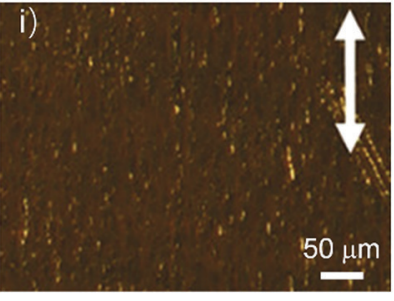

m)

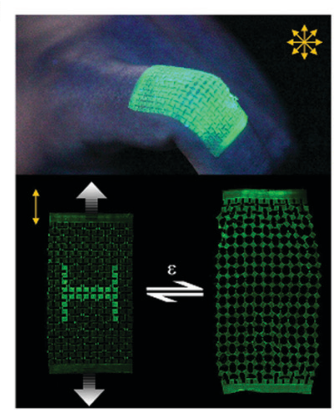

Fig. 4 Anisotropy of the optical properties in ordered NW films. (a) The reflected light optical microscopy image of aligned $3.7 \mu \mathrm{m}$ Au NWs. (b) The experimental transmission spectra of TM and TE polarized light. (c) The schematic of the dual electrode reconfigurable polarizer. The reflected light optical microscopy image of the initial (d) and reconfigurable (e) Au NW film. (f) Preparation process of the ordered NW film. (g) Transmission of light with polarization parallel or perpendicular to the alignment direction of the NW film. ( $\mathrm{h}$ and i) Mapping of fluorescence polarization in the ordered CdSe QR film. (j) Polarized emission spectrum of the printed NW composite. (k) Fluorescence polarization picture of dual polarized light mode. (l and m) Visualization of strain based on the printed NW device. (a-e) Reprinted with permission from ref. 68. Copyright 2017 Wiley. (f-i) Reprinted with permission from ref. 69. Copyright 2019 Wiley. (j-m) Reprinted with permission from ref. 72 . Copyright 2019 American Association for the advancement of Science.

$\mathrm{Cu}$ nanoparticles deposited only on the aligned Ag NWs under electrochemical deposition, resulting in a film with anisotropic wetting behavior arising from the continuous three-phase contact line in the parallel direction ( $x$-axis direction).

Assembly can also influence NW/NR semiconductor electrical properties. For typical semiconductor NWs, an ordered structure promotes the migration of electrons and holes, which is critically important for NW field-effect transistors (FETs) and sensors with fast response. ${ }^{60-62}$ As carried out by Peng and co-workers, a highly dense orientated carbon nanotube (CNT) array was assembled by a directional shrinking transfer method, by shrinking a solutionderived CNT film onto a stretched polydimethylsiloxane (PDMS) film. ${ }^{63}$ All the CNTs showed ordered orientation which was parallel to the shrink direction. The aligned CNT device showed distinct electronic properties in the length and radial direction. In the longitudinal direction, the CNT FET showed higher mobility and on-current density than the radial orientation, which was derived from the faster carrier transfer along with the ordered CNT networks.

\subsection{Anisotropy of optical properties}

Aligned 1D nanomaterial arrays show a polarization effect similar to a metal grating polarizer. ${ }^{64}$ Generally, a flawless grating polarizer needs a periodic line construction with a periodic width less than the wavelength to obtain only zeroorder diffraction for polarization. ${ }^{65}$ An ordered film polarizer could easily reach a minimal periodic width to modulate short wavelength electromagnetic waves (EMW) without precision micro-nano processing. An NW polarizer reflects or absorbs the EMW with an electric field component incident parallel to the wires (TE) due to the excited vibration of free electrons. The electric field component of EMW with perpendicular incidence (TM) can creep across the polarizer without reflecting. This anisotropic optical property derives from the uniform orientation of the assembled 1D nanomaterial structure. Specifically, many noble metal NWs have localized surface plasmon resonance (LSPR) in the visible wavelength. ${ }^{66,67}$ When the incident light wavelength is close to the transverse LSPR peak position, the TM light is absorbed dramatically, which selectively passes 
TE rather than TM light. This aligned film polarization effect can modulate the optical properties, including the transmittance, absorption, and photoluminescence, which can be used for optical storage, encryption, sensing, and full-color displays.

Keating group reported a reconfigurable NW polarizer using the assembly of silica-coated Au NWs. ${ }^{68} \mathrm{Au}$ NWs with different lengths were aligned by a high-frequency electric field assembly method. As shown in Fig. 4a, all the NWs showed uniform orientation along the field by dipole interactions. With the length of the NWs enlarged, the NW film formed more open lattices. The ordered NW film showed broadband light polarizer capabilities in the infrared band (Fig. 4b). The lattice selectively transmitted infrared TM light. To obtain a NW polarizer device with reconfigurability, the authors fabricated two interdigitated electrodes with a perpendicular configuration (Fig. 4c). The electrodes were separated by an adhesive spacer containing NW solution. This device showed two polarization directions which depended on the switching direction of the electric field. The orientation of the NWs could be changed by electrifying the two electrodes in turn as shown in Fig. 4d and e.

A UV light polarizer can also be fabricated by assembling a NW structure. Wang demonstrated a flexible polarizer based on the assembly of sub-1 nm GdOOH NWs. ${ }^{69}$ In this work, the aligned structure of the NWs was fabricated by the wetspinning method as shown in Fig. 4f. A uniform NW gel film was prepared by injecting NW-octane solution into a coagulation bath. The NWs aligned along the extrusion channel and packed further due to solvent exchange. The NW film showed anisotropic UV light transmission of polarization (Fig. 4g). When the light was vibrating perpendicular to the orientation of the NW film, high transmittance could be reached. The high anisotropy of the NW film indicated that the films could be used for polarizers. Interestingly, the authors integrated this NW film with fluorescent quantum rods (QRs), such as CdSe QRs. The composite films emitted polarized fluorescence with the polarization parallel to the orientation direction of the NW film (Fig. 4h and i).

Not only can the anisotropic transmittance be regulated by aligned NW films, but the emittance of photoluminescence of NWs could also be anisotropic by the alignment effect. ${ }^{70,71}$ When incident light with no polarization illuminates the arranged NW array, TE light is selectively absorbed. The anisotropic light absorption causes anisotropic light emission by photoluminescence. For example, an arranged colloidal perovskite NW array could be applied in polarized emission devices. Lewis et al. introduced programmable polarization anisotropy using cesium lead halide perovskite NWs. ${ }^{72}$ They developed a nanocomposite ink consisting of NWs and a polystyrene-polyisoprene-polystyrene block copolymer matrix for 3D-printing. The NWs were highly aligned along the print direction in the polymers induced by shear-induced alignment. The nanocomposites showed highly anisotropic emission due to the alignment effect. The aligned NW film had an emission polarization ratio of $0.48 \pm 0.02$ with one polarizer, which reached $0.71 \pm 0.03$ using a pair of polarizers (Fig. 4j). With
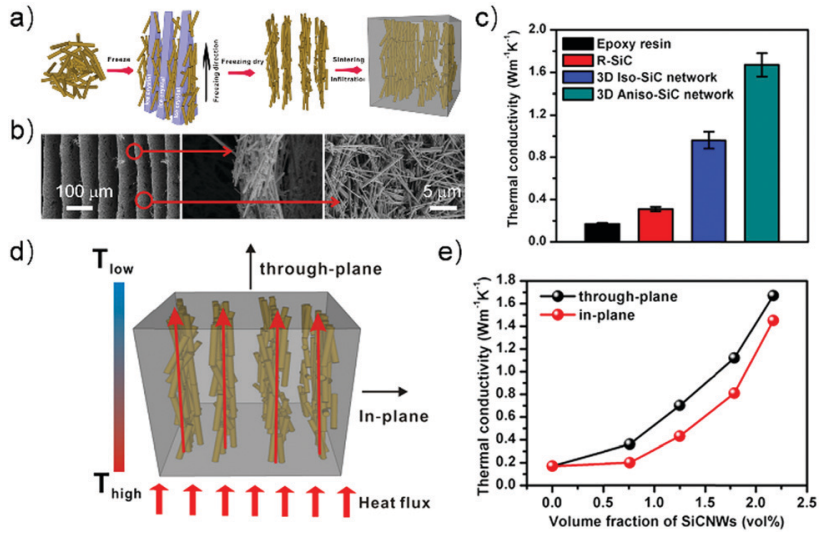

Fig. 5 Anisotropy of the thermal conduction properties in ordered NW composites. (a) Schematic illustration of fabricating SiC NW networks through freeze casting. (b) SEM of the layer structure with the SiC NW network. (c) Thermal conductivity of 3D SiC NW/epoxy composites. (d) Schematic of the anisotropic thermal conductivity in the throughplane and in-plane direction. (e) The influence of the SiCMW volume on the anisotropic thermal conductivity. $(a-e)$ Reprinted with permission from ref. 81. Copyright 2018 American Chemical Society.

the operability and designability of 3D printing, an Escherinspired image was created in which the different regions had different orientations. Under no polarization, the film showed all pictures. But when horizontal or vertical polarization was used, only half of the pattern emerges (Fig. 4k). This polarized emission phenomenon could also be used for mechano-optical metamaterials as shown in Fig. $4 \mathrm{l}$ and $\mathrm{m}$. Under tension, the flexible substrate containing printed NWs deformed and rotated, which meant that the NW orientation changed with strain. Once the NW film rotated, the polarized emission light strength changed following sine wave rules, which could be easily observed by eye.

\subsection{Anisotropy of thermal conductive properties}

Efficient thermal management materials have become one of the most fascinating materials for many applications. ${ }^{73,74} 1 \mathrm{D}$ nanomaterials are often used as fillers in polymer composites to elevate the thermal conductivity, such as CNTs, ${ }^{75} \mathrm{BN} \mathrm{NTs},{ }^{76}$ and so on. Due to the higher thermal conductivity of $1 \mathrm{D}$ nanomaterials, heat transport is fast along the network. The structure of the 1D nanomaterial network in composites influences the heat transfer efficiency heavily. ${ }^{77-80}$ With an ordered configuration, shorter heat transfer pathways along the thermal conductor form in the composites. Energy transport occurs along the 1D nanomaterials array with the shortest pathways and least junctions. The oriented heat transfer network brings an anisotropic thermal property in the parallel direction of $1 \mathrm{D}$ nanomaterials and the perpendicular orientation.

Wong group proposed a strategy to obtain a SiC microwireepoxy resin composite material by the freeze casting technique (Fig. 5a). ${ }^{81}$ In the freezing process, the ice crystals separated the NWs and restricted the degree of freedom in the direction of the ice growth. The NWs were assembled by the freeze casting technique to form a vertical orientation (Fig. 5b). At a low SiC 
NW loading, the composites showed a gradually improving thermal conductivity with higher NW loading. However, the thermal conductivity of the ordered structure composite showed a remarkable improvement compared to the random cases, which was related to the continuous phonon pathway in the aligned samples. The disordered NW sample could only reach $0.35 \mathrm{~W} \mathrm{~m}^{-1} \mathrm{~K}^{-1}$, which was a quarter of the aligned configuration (Fig. 5c). Due to the ice extrusion, the NWs were all distributed in a regional plane. So, the material also had an anisotropic heat transfer property between the through-plane and in-plane direction as shown in Fig. 5d. Feng and coworkers used a similar method to fabricate polyvinyl chloride (PVC) foam plates with vertically aligned SiC NW networks. The aligned sample with only 1.32 vol\% of SiC NWs had a thermal conductivity of $0.62 \mathrm{~W}(\mathrm{~m} \mathrm{~K})^{-1}$, which was 2.3 -fold that of the random case (Fig. 5e).

Anisotropic thermal conductivity can also be observed in a CNT composite material. As performed by Kenneth E. Goodson, aligned CNTs brought anisotropic thermal conductivity in CNT-epoxy composites. ${ }^{82}$ The thermal conductivity for the axial direction was much greater than the transverse direction in the aligned CNT-epoxy composites, while the disordered CNT sample showed isotropic thermal conductivity. The authors attribute the anisotropy to the aligned CNTs forming percolation networks for more effective heat transport.

\subsection{Anisotropy of magnetic properties}

A magnetic medium shows magnetophoretic alignment behavior under a magnetic field caused by a magnetic dipole moment. For magnetic 1D nanomaterials, head-to-tail orientation could form under the stimulation of a magnetic field. NWs often grow with a specific orientation, which means with ordered assembly all the NWs show the same orientation in the assembly direction. A magnetic material shows different susceptibility with different orientations. It means that with an aligned NW array, the magnetization energy is anisotropic. ${ }^{83}$ Also, the demagnetizing energy inside the NWs is closely related to the morphology, which induces strong shape anisotropy of demagnetization. It is also worth mentioning that the magnetic dipolar interactions between adjacent NWs would be larger with a close-packed structure when perfectly assembled. These three factors cause the magnetic anisotropic effects of ordered NW arrays.

Our group demonstrated the self-assembly of $\mathrm{SiO}_{2}$-coated $\mathrm{Fe}_{3} \mathrm{O}_{4} @ \mathrm{Ag}$ NWs under a magnetic field. ${ }^{84}$ Fig. 6a reveals the assembly procedure with solution evaporation and the magnetophoretic alignment effect. All the NWs showed the same orientation along the magnetic field with orientation parameter $S$ close to 1 . This aligned NW film had strong magnetic anisotropy. As shown in Fig. 6b, the field dependence of magnetization along the orientation direction was much higher than the perpendicular direction with a ratio of 2.01. We also performed an electron paramagnetic resonance (EPR) test to reveal the magnetic anisotropy. For the ordered sample, the resonance shifted by a small degree in the parallel and perpendicular direction (Fig. 6c), which indicated the angular

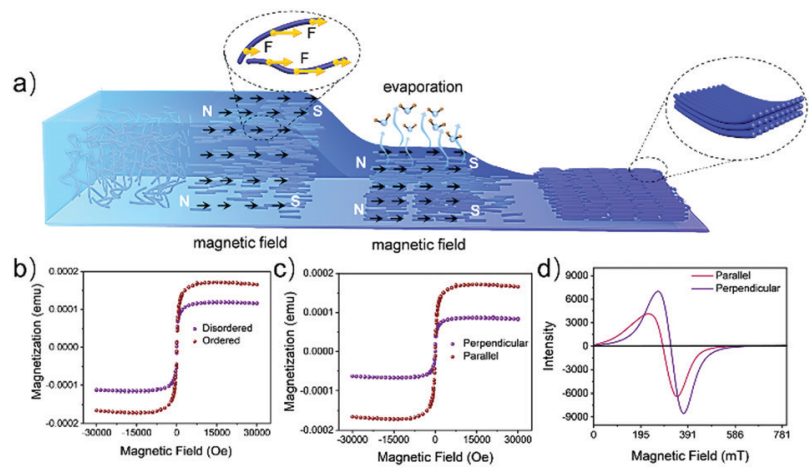

Fig. 6 Anisotropy of magnetic properties in ordered NW films. (a) Schematic illustration of the magnetic assembly process. (b) The magnetization of the ordered NW film with the magnetic field parallel and perpendicular to the orientation. The EPR spectra of the aligned (c) and random (d) sample in the parallel and perpendicular direction. $(a-d)$ Reprinted with permission from ref. 84. Copyright 2020 Wiley.

dependence effect of the aligned sample. Meanwhile, in the random case, the EPR showed no shift with different magnetic field angles (Fig. 6d), which revealed the magnetic isotropy in the random NW assembly.

Polymeric films could also show magnetic anisotropy with embedded ordered magnetic 1D nanomaterials. The Athanassiou group presented magnetic poly-(methyl methacrylate) (PMMA)-CNT nanocomposite films with typical magnetic anisotropy. ${ }^{85}$ Although CNTs have extremely low magnetic susceptibility, the authors prepared magnetic CNTs by mixing CNTs with $\mathrm{Fe}_{3} \mathrm{O}_{4}$ nanoparticles. Then the CNTs were assembled under a magnetic field with aligned structure and cured with PMMA. This nanocomposite film showed clear magnetic anisotropy.

\subsection{Anisotropy of strain sensing properties}

1D nanomaterial strain sensors have attracted tremendous attention due to their application in wearable devices. ${ }^{86-88} 1 \mathrm{D}$ nanomaterials with excellent electrical and mechanical properties are used in flexible strain sensors, such as Ag NWs and CNTs. Strain has both a value and a direction as a vector. In general, one NW strain sensor shows the same response under omnidirectional strain with a disordered configuration. This means that random NW strain sensors could only measure the strain value with no direction recognition. To obtain a multidirectional strain sensor, the response under the same strain in different directions must be different. NWs with an oriented structure provide a promising strategy for the realization of multidirectional sensing. Under the same strain, the percolation network changes variously in different directions, resulting in various gauge factors under multidirectional tension. This anisotropy of strain sensing could be applied in detecting complex strain conditions.

$\mathrm{Xu}$ and co-workers reported a strain sensor with the anisotropic property of multiaxial detection, which was fabricated by the carbonization of common crepe paper. ${ }^{89}$ Fig. 7a demonstrates that the special anisotropic structure of crepe paper 
a)

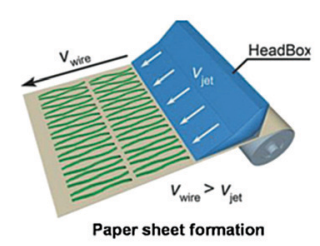

c)

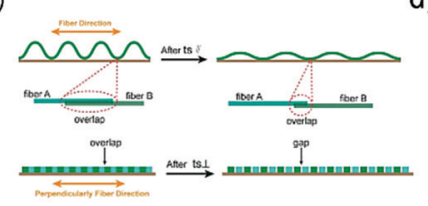

e)

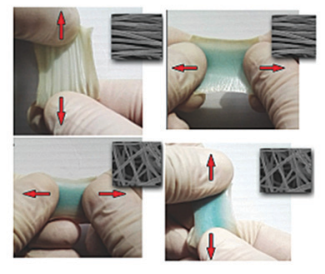

b)

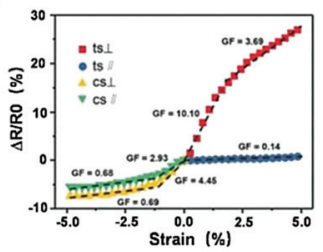

d)

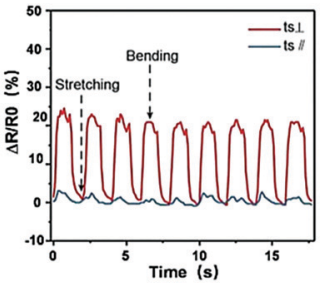

f)

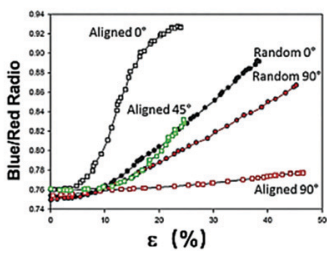

Fig. 7 Anisotropic strain sensing with aligned NW structures. (a) Schematic of fabrication of the cellulose film with aligned fibers. (b) The orientation of the fibers resulted in the anisotropic sensitivity of the sensor. (c) Schematic of the anisotropic performance and sensing depending on the fiber arrangement mechanism. (d) The anisotropic response of the strain sensor in different strain directions. (e) Images of the anisotropic allochroic performance attributed to the direction of fibers. (f) The allochroic comparison of the samples with a different direction of fibers and degree of order. (a-d) Reprinted with permission from ref. 89. Copyright 2018 Wiley. (e and f) Reprinted with permission from ref. 93. Copyright 2018 Wiley.

could be well saved after carbonization. This structure resulted in different change of conductivity for bending directions parallel to the array direction or not (Fig. 7b). Fig. 7c shows the deformation mechanism of the fibers under strain. The overlap of conductive fibers still existed under parallel strain. However, the connections were broken under vertical strain. The varied response to strain originated from varying degrees of breakdown of the percolation network. With the above features, this crepe paper could detect the strain sensitively along the defined direction (Fig. 7d). Only when the stress was perpendicular to the fibers did the sensors show a huge response due to the disconnection between conductive fibers. Moreover, by integrating two sensors with a vertical orientation structure, both the magnitude and direction can be read for multidimensional sensors.

A special sensor with an anisotropic piezoresistive effect can also be realized by electrospinning too. Kim et al. presented a multidimensional mechanical sensor fabricated through an assembly method of electrospinning for anisotropic carbon NWs ${ }^{90}$ which possessed excellent properties with a parallel force selectivity of 3.84, and favorable stability over 2500 cycles. This sensor could realize the measurement of multiaxis strain since the resistance of two carbon NW layers changes to varying degrees depend on the direction of the strain. Parallel strain could break the joints of carbon fibers and increase the resistance.

Conversely, the connections of the network were maintained well under vertical strain, so the sensor was not sensitive to perpendicular forces.

Recently, visualization of strain and pressure has attracted tremendous attention due to the readability of information. ${ }^{91,92}$ Visual strain sensors are often constructed from mechanochromic materials. But in most reports the materials uniformly distribute in the matrix with the same discoloration under strain without direction recognition. Assembly can bring anisotropic discoloration for both a level and direction strain response. Gualandi and co-workers reported a real-time mechanochromic strain sensor with an anisotropic response composed of mechanochromic P(SP-alt-C10) nanofibers and PDMS, in which the mechanochromic nanofibers were fabricated through the electrospinning method. ${ }^{93}$ As shown in Fig. 7e, the aligned nanofibers showed an anisotropic discoloration degree since the strain on the fibers was different in the two directions. Also, compared with films from the solvent evaporation method, the bundle of nanofibers exhibited excellent mechanochromic properties. As shown in Fig. 7f, the orientated $\mathrm{P}(\mathrm{SP}-$ alt-C10) nanofibers with larger deformation exhibited darker color than the random cases and stress parallel to the direction of the fibers could result in variation of the color, which indicated that the color transformation was related to the orientation of the mechanochromic fibers.

\section{Performance enhancement of ordered 1D nanomaterial-based assemblies}

1D nanomaterials with ordered structure exhibit superior properties compared to random samples. ${ }^{19}$ The assembly processes not only change the microstructure but also the microphysical and chemical fields. Also, ordered assembly shows designability due to the procedural operations, which means that the architecture is programmable for complicated devices. The gains from ordered assembly can be briefly categorized as follows:

(a) Ordered assembly forms dense and straight networks for more efficient transportation of electrons, ions, and phonons, which improves the performance of ordered nanodevices compared to disordered pieces.

(b) 1D nanomaterials with high crystallinity have certain crystal orientation along the radial or axial direction. When the physical properties of materials are dependent on the crystal orientation (such as the Seebeck factor, the piezoelectric coefficient), the process of NW assembly will optimize these physical properties along the optimal crystal orientation.

(c) The periodic structure of aligned 1D nanomaterials forms a uniform and periodic micro-environment for homogeneous properties of nanosystems.

(d) The number of layers, location, density, and types of 1D nanomaterials could be easily defined by ordered assembly, which are key to designing accurate and large-scale devices. 
a)

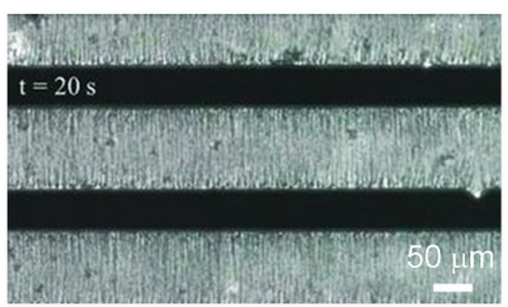

b)

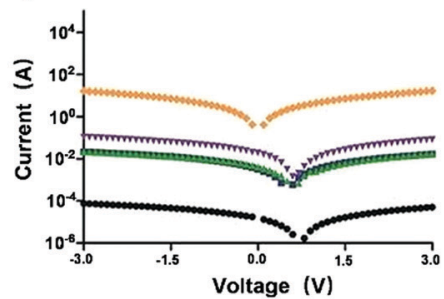

g) d)

e)

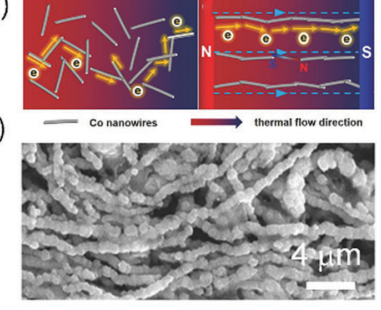

f) 8000

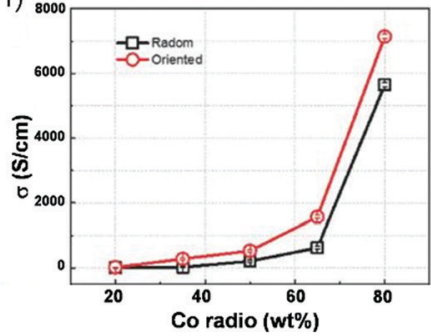

c)

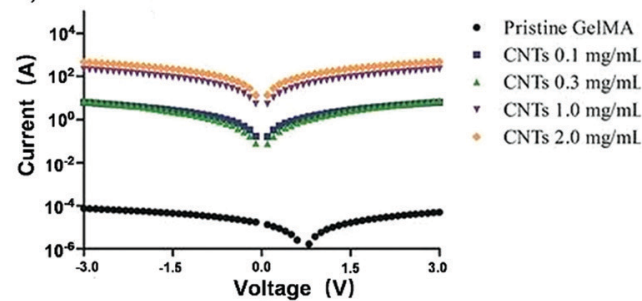

h)

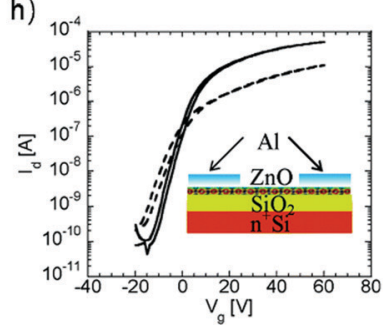

Fig. 8 Improvement of the electrical conductivity of aligned NW nanostructures. (a) Image of ordered CNTs under dielectrophoresis force. Currentvoltage curves for GelMA-random CNT gels (b) and GelMA-aligned CNT gels (c) for different concentrations of CNTs. (d) The schematic of electron transport of ordered and random Co NW/PVDF nanocomposites. (e) SEM image of the aligned Co NW/PVDF nanocomposites. The function of the electrical conductivity ( $f$ ) and power factor $\left(\mathrm{g}\right.$ ) of ordered and random nanocomposites. (h) $I_{\mathrm{d}}-V_{\mathrm{g}}$ curves for OCTA-ZnO nanorods (solid line) and BUTA$\mathrm{ZnO}$ nanorods (dashed line). (a-c) Reprinted with permission from ref. 103. Copyright 2013 Wiley. (d-g) Reprinted with permission from ref. 106. Copyright 2018 Wiley. (h) Reprinted with permission from ref. 107. Copyright 2006 American Chemical Society.

(e) The process of ordered assembly is repeatable with properties and structural stability for widespread commercial applications of 1D nanomaterials.

In this section, we will review the performance enhancement effect of ordered 1D nanomaterial assemblies. Recent developments will be introduced in detail, including electric devices, large scale integrated circuits, strain sensors, nanogenerators, structural color (SC) films, structural materials, and energy fields.

\subsection{Assembly of NWs for electrical conductors}

1D nanomaterial-based conductor devices receive more attention due to flexibility and integrability with other devices. In most NW-based electric devices, the NW conductors work as the cornerstone..$^{94-98}$ For a conductor with the same number of NWs, more overlapping of the NW network means lower resistance. Disordered NWs often have an open gap between NWs, which brings hindrance for the transfer of carriers. Assembly of NWs can improve the conductivity of the NW network with a more strongly conducting percolation network. The assembly technique packs the NWs into a dense array and increases the overlapping junction between NWs. This assembly effect could instruct the design of electrical devices, such as flexible conductors, NW field-effect transistors, and photodetectors. ${ }^{60,99-102}$

Matsue group has reported the fabrication of ordered CNTs in gelatin methacrylate (GelMA) hydrogels by dielectrophoresis (DEP). ${ }^{103}$ The CNTs dispersed in the GelMA prepolymer generated a dipole moment under the stimulation of an AC electric field and then the CNTs arranged along the direction of the electric field as shown in Fig. 8a. Subsequently, the prepolymer was polymerized under irradiation with ultraviolet light to fix the aligned CNTs. Compared with random CNTs in GelMA hydrogels, the electrical conductivity of the GelMA-CNT hydrogels increased about two orders of magnitude when they were arranged in order (Fig. $8 \mathrm{~b}$ and c). The reason for the higher conductivity is that the ordered GelMA-CNT hydrogel conductive paths form straight channels, which promotes the transfer of electrons.

The improved conductivity of a NW assembly shows vast potential in NW thermoelectric materials since the thermoelectric figure of merit is proportional to the conductivity coefficient. ${ }^{104,105}$ This strategy was presented by the Liang group to enhance the thermoelectric properties of organicinorganic composites containing aligned Co NWs and poly(vinylidene fluoride) (PVDF). ${ }^{106}$ The composite was constructed by mixing Co NWs and PVDF in DMF and selectively assembling Co NWs by applying a magnetic field. As shown in Fig. 8d, a lot of the random Co NWs were not overlapping with each other. However, the ordered Co NWs were overlapping with each other along the uniform direction (Fig. 8e), forming a percolation network to obtain higher conductivity. The aligned Co NW/PVDF film was combined with a PEDOT:PSS to fabricate a flexible thermoelectric device, which showed higher output power and output voltage than the disordered sample at the same temperature gradient (Fig. $8 \mathrm{f}$ and $\mathrm{g}$ ). This work demonstrates the important role of assembly in improving the performance of flexible thermoelectric devices.

Assembly can also improve the semiconductor FET performance by structure control. In NW semiconductor systems, electrons and holes move quickly along the aligned NWs with the least junction jumping. The larger mobility promotes advanced FET and photodetector devices with low switching delay time. Liu et al. demonstrated an aligned ZnO NR film 
prepared by spin-coating nanorod solution on a $\mathrm{SiO}_{2} / \mathrm{Si}$ substrate treated with hexamethyldisilazane (HMDS). ${ }^{107}$ With solution evaporation, the ZnO NRs were aligned due to the radial fluid flow. Furthermore, the surfactant became the main governing factor of the assembly morphology. The authors used butylamine (BUTA) or octylamine (OCTA) as a ligand for different assembly processes. In the OCTA-ZnO film, the nanorods were arranged in a uniaxial parallel direction in a large domain on the film plane. In contrast, in the BUTA-ZnO film, the uniaxial parallel arrangement was only in smaller areas of the nanorod film. Therefore, the field-effect transistor prepared from self-assembled nanorod films showed markedly enhanced electrical transport performance. As shown in Fig. 8h, the performance of thin-film transistors (TFT) with OCTA-ZnO nanorods was excellent compared to the film with BUTA-ZnO nanorods. The authors attribute this improvement to the large domain size of ordered nanorods with faster charge transfer.

\subsection{Assembly of NWs for large-scale integrated circuits}

1D nanomaterials have exceptional electronic properties, such as high carrier mobility, excellent switching characteristics, and superior photoelectric effects, which can be designed to fabricate complicated electronic devices including integrated circuits, microsensors, and optoelectronics. ${ }^{108-114}$ Often, 1D nanomaterials are aligned in a uniform direction and form the desired pattern by assembly such as the LS method, contact printing, and self-assembly. Compared to crude random assembly, the superiorities of precise assembly with definite architecture can be briefly summarized as follows:

(a) The charge carrier mobility is highest along the NW longitudinal direction with the least junction jumping, which is crucial for high-speed processors.

(b) Assembly ensures a high density of NWs for elevating the transistor current output to compete with silicon-based devices.

(c) The aligned NWs avoid crossing defects for devices based on single NWs or the mutual interference between two adjacent NW array transistors.

(d) Homogeneous distribution of NWs in a large area can improve the device-to-device uniformity, even when the properties of each NW are different.

(e) Precise control of the type of NWs in the designated location is a prerequisite for fabricating CMOS microprocessors. Also, the controllable integration of multiple NWs promotes the development of multifunctional micro/nano-sensors.

More recently, Lieber and co-workers reported programmable NW circuits base on a Ge/Si core/shell NW assembly. ${ }^{115}$ The NWs were synthesized by a nanocluster-catalysed methodology and axially aligned by lubricant-assisted contact printing as shown in Fig. 9a. The gate response of the single NW-FET showed stable conductance behaviors under gate voltage control. Also, a coupled multigate device was successfully fabricated by the assembly of two NWs. One NW-FET output was coupled into the gate input of another to obtain programmable NW-FET functions, thus suggesting that the assembly of NWs into a precise architecture was important for NW processors. a)

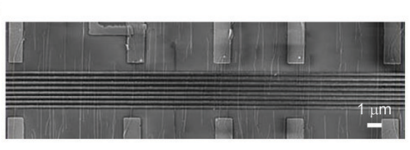

b)

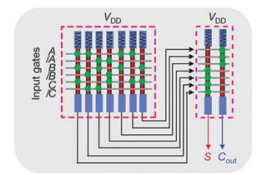

c)

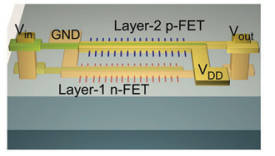

d)

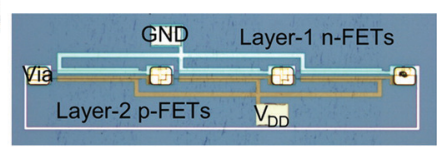

e)
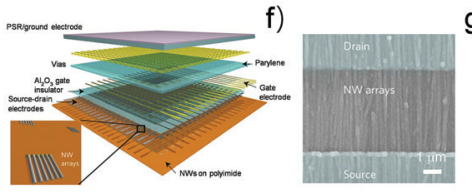

g)

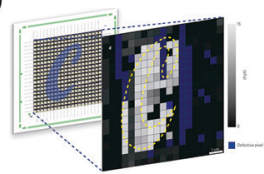

Fig. 9 NW assembly for large-scale integrated circuits. (a) Picture of aligned NWs with the electrode design. (b) Ordered NW network design for a one-bit full-adder. (c and d) Co-assembly of two types of NWs for inverters and ring oscillators. (e) Schematic diagram of the large-scale assembled NW array pressure sensor device. (f) SEM of the aligned $\mathrm{Ge} / \mathrm{Si}$ NW array. (g) Two-dimensional intensity profile of the pixel signals. ( $a$ and b) Reprinted with permission from ref. 114. Copyright 2013 Nature Publishing Group. (c and d) Reprinted with permission from ref. 118. Copyright 2009 National Academy of Sciences. (e-g) Reprinted with permission from ref. 119. Copyright 2010 Nature Publishing Group.

The authors further developed a one-bit full-adder (Fig. 9b), a full subtractor, and a D latch circuit with the same NW architecture with different circuit designs. The aligned NWs ensure programmable circuit design for complicated logic functions and provide the possibility of single NW-FET integration. Qing Cao demonstrated the LS technique to obtain dense and full surface covered CNT field-effect transistors. ${ }^{116}$ By compressing and aligning CNTs on an air-liquid surface, the CNTs formed a dense film, which reached a CNT density of 500 tubes per $\mathrm{m}$. With more CNTs, the current density could reach $120 \mathrm{~mA} \mathrm{~m}^{-1}$ to compete with silicon-based devices.

In the theory of modern CMOS technology, only one type of NWs restricts the realization of complex logical operation. The integration of two types of NWs of p-type and n-type is the fundament of a NW-based CMOS device. ${ }^{35,117}$ The Liber group also reported alignment of two types of NWs on one substrate to construct CMOS inverters and ring oscillators (Fig. 9c and d). ${ }^{118}$ They used layer-by-layer assembly of Ge/Si p-FETs and InAs n-FETs to fabricate an integrated circuit. The NWs were prepared by CVD and transferred to the device substrate by a shear printing process. The substrate was patterned by photolithography to predefine the area in which NWs were deposited. The number of NWs was controlled by the trench width. Integration of two types of NWs was indispensable in nanoelectronics with complex functions. With precisely placing the various NWs, the logic circuits could be interconnected and this process was coupled with modern Si-CMOS technology.

For artificial skin, large-scale integration of sensor components with an active-matrix is a top priority. Using NW arrays as sensors is a good strategy due to the excellent response under stimulation. Javey group proposed NW active-matrix circuitry for pressure macroscale sensors. ${ }^{119} \mathrm{Ge} / \mathrm{Si}$ core/shell NW parallel 
a)

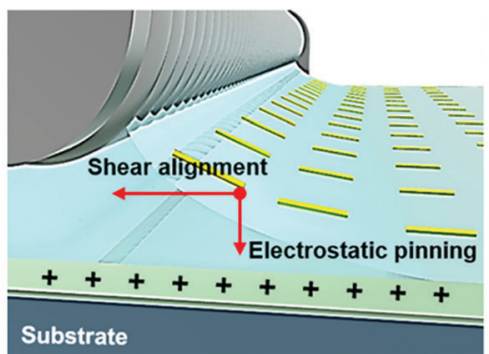

d)

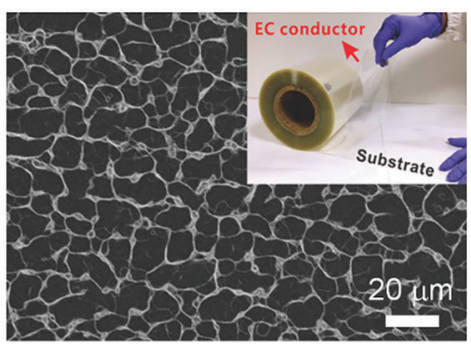

h)

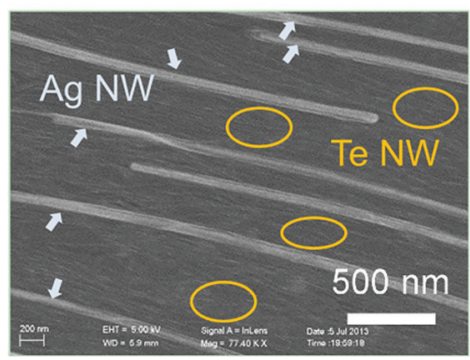

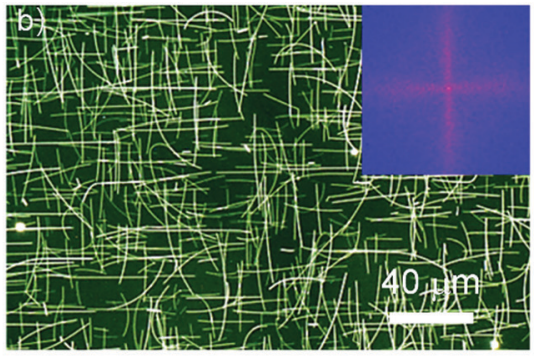

e)

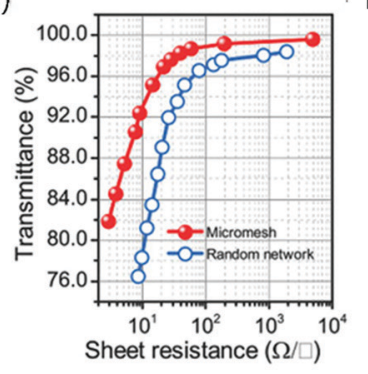

i)

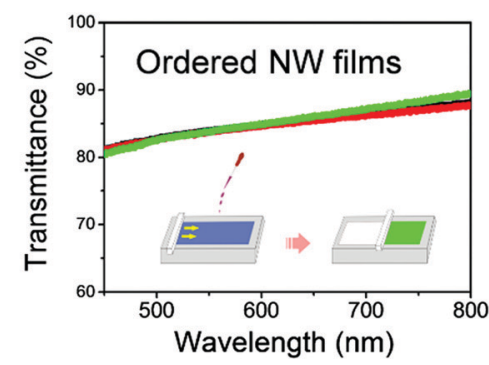

c) f)

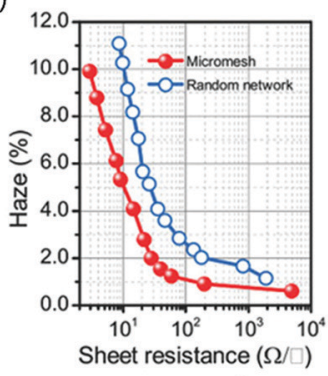

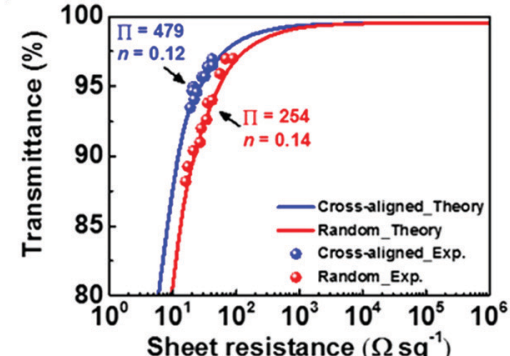

g)

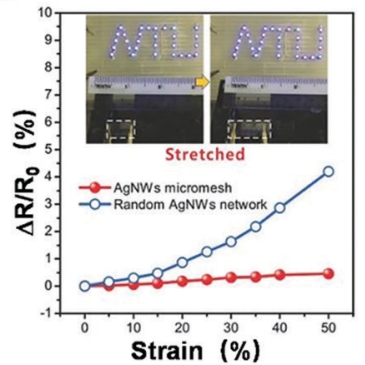

j)

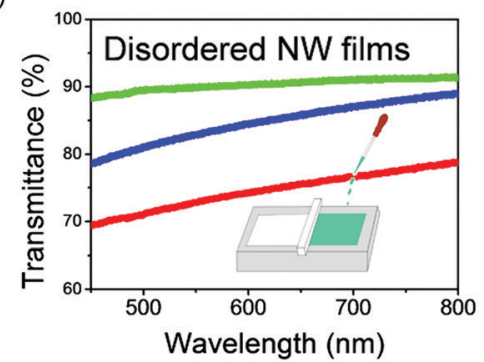

Fig. 10 Aligned NWs for optimizing the performance of TCEs. (a) The alignment process of Ag NWs during the bar-coating assembly. (b) Dark-field optical microscope image of the cross-aligned Ag NW array. The inset is the fast Fourier transform (FFT) analysis of the optical micrograph. (c) $R_{\mathrm{s}}$ versus optical transmittance at $550 \mathrm{~nm}$ for the cross-aligned and random Ag NW network. (d) SEM of the Ag NW micromesh with EC substrate. The inset is a photo of the EC conductor with a PET roll. Transmittance and haze of the micromesh (e) and random networks (f) as a function of $R_{\mathrm{S}}$, respectively. (g) $\Delta R / R_{0}$ versus uniaxial strain for a stretchable Ag NW micromesh conductor. The insets are photos for demonstrating the stretched conductor that kept the LEDs lit. (h) SEM image of NW co-assemblies, monolayers of Ag and Te NWs. Optical transmission spectra of the ordered (i) Ag NW film and disordered (j) NW film. (a-c) Reprinted with permission from ref. 25. Copyright 2017 American Chemical Society. (d-g) Reprinted with permission from ref. 24. Copyright 2018 Wiley. (h-j) Reprinted with permission from ref. 125. Copyright 2014 Wiley.

arrays were transferred to a polyimide substrate by a contactprinting method (Fig. 9e). The location of the NW array was predefined by lithography. Each NW array worked as a pressure sensor with the same performance owing to the uniform distribution by the large-scale assembly. As shown in Fig. 9f, in a $7 \times 7 \mathrm{~cm}^{2}$ area, a $19 \times 18$ pixel pressure sensor array could be integrated. The sensor matrix can measure pressure in the large area and form pressure mapping (Fig. 9g), which was analogous to human skin.

\subsection{Assembly of NWs for transparent conductive electrodes}

1D nanomaterials including $\mathrm{Ag}$ NWs, ${ }^{120} \mathrm{Cu} \mathrm{NWs}{ }^{121}$ and CNTs $^{122}$ show great potential in TCEs due to the ultrahigh conductance and ultrafine size. ${ }^{123}$ Especially, Ag NW TCEs have had actual applications in commercial display screens. In primary investigation, a NW TCE is often fabricated by spraying or dip-coating without structure control. ${ }^{123}$ These crude processes often cause an inhomogeneous distribution of NWs without predictable morphology, which reduces the transmittance and conductivity. This means that designing the NW network microstructure plays a critical role in producible TCEs with high performance. With a design, the NWs distribute homogeneously on the large-scale substrate to ensure high performance of the TCEs.

Ko and co-workers reported a simple, fast, and large-scale bar-coating assembly strategy for the fabrication of TCEs based on a cross-aligned Ag NW network with a sheet resistance of $21.0 \Omega \mathrm{sq}^{-1}$ at $95.0 \%$ optical transmittance. ${ }^{25}$ Fig. 10a shows a photograph of the bar-coating assembly process on a large polyethylene terephthalate (PET) substrate $\left(20 \times 20 \mathrm{~cm}^{2}\right)$. During the bar-coating assembly, the interaction between shear-induced hydrodynamic and electrostatic forces caused the formation of a highly aligned Ag NW network (Fig. 10b), which had lower sheet resistance, lower percolation threshold, and better transparency (Fig. 10c) than the disordered $\mathrm{Ag}$ NW network. Furthermore, the assembly sample had more uniform sheet resistance and optical transmittance than the random cases, induced by the homogeneous distribution of $\mathrm{Ag}$ NWs. 
The Ko group also provided a capillary printing technique to precisely control the alignment and the percolation behavior of $\mathrm{Ag}$ NW networks which were used for polymer light-emitting diodes (PLEDs). ${ }^{124}$ During the capillary printing process, the Ag NWs were aligned by the solvent-evaporation-induced capillary force at the solid-liquid-vapor contact line. Compared with PLEDs made from random Ag NWs, these PLEDs which used aligned Ag NWs showed a 30\% enhanced maximum luminance (33068 $\mathrm{cd} \mathrm{m}^{-2}$ ) and a higher luminance efficiency (14.25 $\mathrm{cd} \mathrm{A}^{-1}$ ). These advantages were attributed to the low electrical percolation threshold of aligned $\mathrm{Ag}$ NWs, which were beneficial for the achievement of lower resistance and higher transmittance compared to random ones.

Furthermore, the self-assembly method can also be applied in TCE fabrication. Lee et al. provided a spray-assisted selfassembly process to form a scalable $\mathrm{Ag}$ NW bundle micromesh with controllable ring size $(4-45 \mu \mathrm{m})$ that could be easily realized on various polymer substrates, resulting in it being transferable to many transparent and deformable substrates. ${ }^{24}$ Fig. 10d showed the ordered configuration of the $\mathrm{Ag} \mathrm{NW}$ network. Compared to the conventional random Ag NW network, this Ag NW bundle micromesh had explicit conductive paths and reduced optical extinction cross-sections, requiring lower coverage of the whole substrate with NWs. These advantages led to higher transparency (transmittance of 97\%) and lower haze $(2.6 \%)$ with a lower junction resistance of $25 \Omega \mathrm{sq}^{-1}$ (Fig. 10e and f). The assembly improved the mechanical durability greatly due to the arranged and local-dense structure. As shown in Fig. $10 \mathrm{~g}$, the assembly NW micromesh can bear 50\% strain without degradation. However, the random sample showed a $420 \%$ increase in resistance under the same strain.

Co-assembly for fabricating TCEs was first reported by our group. ${ }^{125,126} \mathrm{Ag}$ and Te NWs were co-assembled by the Langmuir-Blodgett technique to fabricate a flexible TCE. The Te NWs were selectively etched to obtain Ag NWs with space. The interspacing could be adjusted by the Ag-Te NW ratio, which means that the sheet resistance and optical transmittance could be regulated. Fig. 10h presents a monolayer of $\mathrm{Ag}$ and Te NWs with uniform orientation. After etching the Te NWs, highly flexible and transparent Ag NW films could be attained. Moreover, comparing the optical transmission spectra of the random and ordered arranged NWs with the same NW density, the ordered NW films were more stable and reproducible (Fig. 10i and j).

\subsection{Assembly of NWs for strain sensors}

Strain sensors act as a transducer which transforms a tensile force signal into an electric signal. ${ }^{127,128} \mathrm{~A}$ 1D nanomaterial network cooperates with flexible and stretchable polymer substrates. When tension is applied, the polymers deform, and the 1D nanomaterials start slipping and separating. ${ }^{129,130}$ In the disconnection mechanism, electrons pass through overlapped 1D nanomaterials, which means that the slipping decreases charge pathways and increases the resistance. Ordered 1D nanomaterial structure can significantly enlarge this effect since nearly all the charge pathways are parallel to the strain. On the contrary, a disordered network transports charges in all directions, which could buffer the disconnection in the tension direction.

As shown in Fig. 11a, Hata and co-workers presented a strain sensor composed of ordered single-walled carbon nanotubes (SWCNTs). ${ }^{131}$ The sensor consisting of oriented SWCNTs exhibited a superior detection property compared with a random SWCNT sensor (Fig. 11b). The aligned SWCNT sensor showed a broad gauge to $300 \%$, while the disordered sample can only stand $5 \%$ deformation. The authors inferred that bundles of ordered SWCNTs could connect the gaps generated in the period of stretching (Fig. 11c), which resulted in the improvement of the sensitivity. Thus, this aligned SWCNT sensor possessed excellent duration and a fast response, and could be applied in the field of detection of human motion such as breath, phonation, and the postures of the wrist. a)

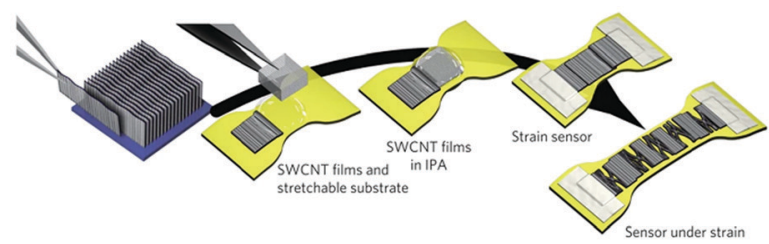

d)

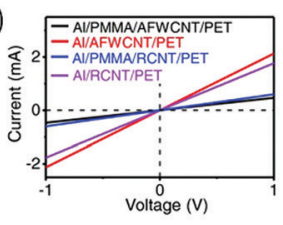

e)

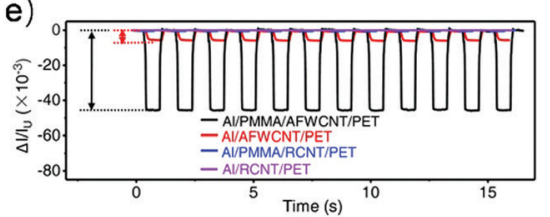

b)

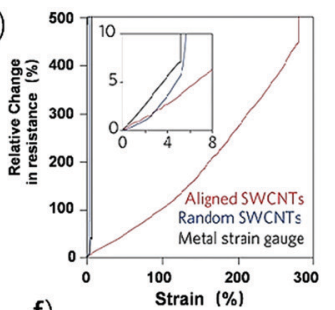

f)

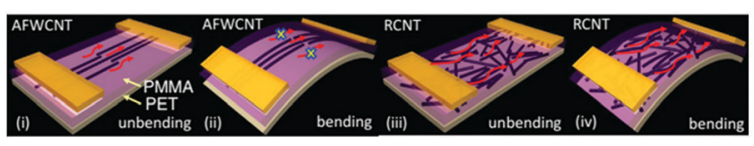

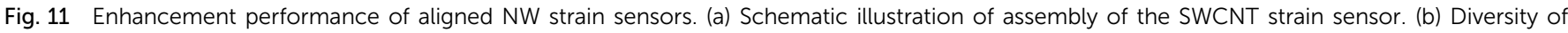

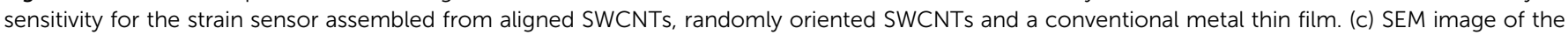

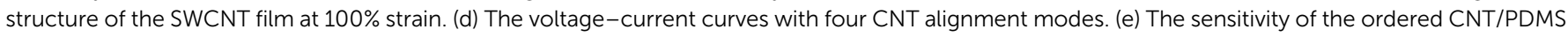

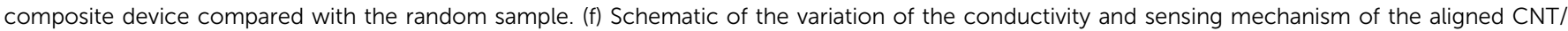

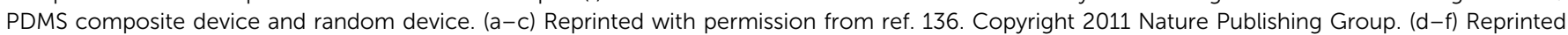
with permission from ref. 137. Copyright 2017 Wiley. 


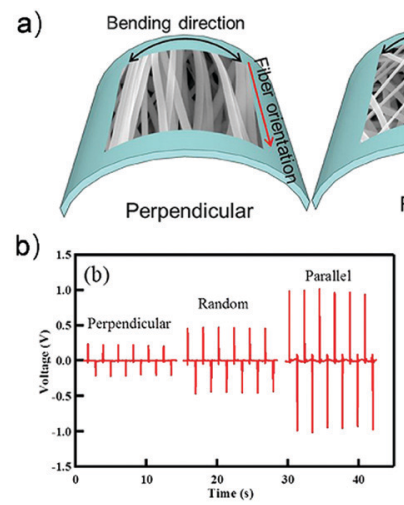

d)

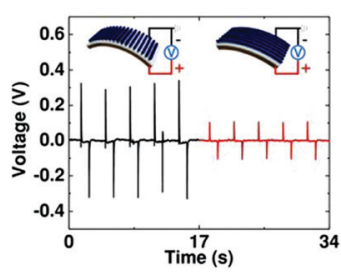

e)

c)
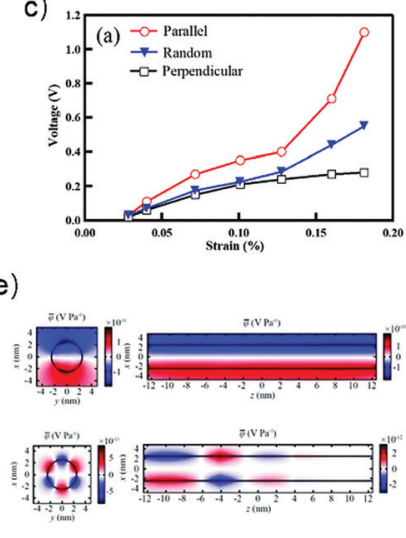

Fig. 12 Elevated piezoelectric effect of the aligned NW structure. (a) Schematic diagram of flexible PZT nanogenerators with different architecture. (b) Output voltage of three different nanogenerators. (c) The relationship of the output voltage with strain with different NW alignment modes. (d) Output voltage along the different orientation of the Te NW film. (e) The piezoelectric potential distribution with the axial and radial strain in Te NWs. $(a-c)$ Reprinted with permission from ref. 142. Copyright 2019 American Chemical Society. ( $d$ and e) Reprinted with permission from ref. 145. Copyright 2013 Wiley.

Recently, this ordered structure has also been applied in bending sensors to obtain a superior sensing effect. Liu et al. reported a strain sensor composed of ordered CNT/PDMS composite devices with anisotropic sensing performance, which embodied excellent sensitivity compared with a sensor composed of random CNTs (Fig. 11d and e). ${ }^{132}$ As shown in Fig. 11f, the authors proposed the percolation model to explain the improved performance of the ordered CNT sensor. The initial joints of orientated CNTs would be interrupted under the tension force, resulting in wide differences of electric conductance. In contrast, the random CNT sensor exhibited conductance that is independent of tension due to that the connections of CNTs always being maintained.

\subsection{Assembly of NWs for nanogenerators}

1D nanomaterials with asymmetric crystal structure are widely used in nanogenerators. ${ }^{133,134}$ Due to the high aspect ratio, 1D nanomaterials have small flexural rigidity and high flexibility, which guarantees large mechanical force-energy conversion efficiency. ${ }^{135,136}$ Compared to the disordered configuration, 1D nanomaterials with uniform orientation can output larger power under the same strain. The assembly effect can be split into two aspects:

(a) When the orientation of the NWs in nanogenerators is in accordance with the direction of strain, it ensures the largest deformation to generate the largest power.

(b) The ordered 1D nanomaterial structure has small internal resistance due to superior percolation, which reduces internal resistance loss of power for larger output power.

Seo et al. reported the fabrication of piezoelectric nanogenerators using lead zirconate titanate (LZT) nanofibers. ${ }^{137}$ To investigate the piezoelectric performance with different fiber stacking arrangements, they manufactured nanofibers by a sol-gel electrospinning process with ordered and disordered models. The nanofibers with disordered allignment can be collected by a mesh-type collector while aligned nanofibers can be fabricated using multipair metal wire collectors (Fig. 12a). The aligned fibers parallel to the binding direction showed the largest piezoelectric voltage (Fig. 12b and c), which was two fold higher than the random sample. The perpendicular sample showed the lowest energy output. The authors proposed that the deformation degree of the LZT fibers was closely related to the assembly morphology. Only when the aligned fibers were parallel with the bending direction were nearly all the fibers under a tension state and the output energy was at the highest level. Furthermore, Henry A. Sodano et al. employed the printing fabrication technique to obtain LZT NW nanogenerators. ${ }^{133}$ All the NWs showed homogeneous orientation along the printing flow by shear-induced alignment. One noteworthy phenomenon is that the power output of the ordered sample was $273 \%$ higher compared to previous cast nanocomposites with random NWs.

$\mathrm{ZnO}$ NWs were the first material used in nanogenerators due to their excellent piezoelectric property along the $c$-axis. A ZnO NW array can only convert pressure to electric energy for a small length and vertical growth mode. To obtain large energy output under bending or tension, horizontal alignment of NWs with multilayers is the key step. For example, the Wang group reported a flexible high-output nanogenerator based on a lateral $\mathrm{ZnO} \mathrm{NW}$ array. ${ }^{138}$ The authors transferred vertically growing $\mathrm{ZnO} \mathrm{NWs}$ to a flexible substrate to form a horizontally aligned array by the sweeping-printing method. The output voltage can be elevated by a series NW network. With the horizontal assembly model, the device generated a peak power density of $\sim 11 \mathrm{~mW} \mathrm{~cm}^{-3}$, which was enough to light up an LED.

Similarly, Wang and co-workers fabricated a high power density piezoelectric nanogenerator by assembling an ultrathin trigonal Te NW array. ${ }^{139}$ Unlike most NW nanogenerators with the strain in the longitudinal direction of the NWs, the nanogenerator of Te NWs was designed with the strain in the radial direction due to the asymmetric crystal structure along this direction. The assembled Te monolayer film showed anisotropic power output under bending. As shown in Fig. 12d, the output voltage and current of NGs with NWs aligned parallel to the bending line were three times higher than the t-Te NWs aligned perpendicularly. The simulation data confirmed that owing to the assembly process, most of the Te NWs were under tensile stress (Fig. 12e) for the maximum power output.

\subsection{Assembly of NWs for structural color devices}

Structural color (SC) of chiral photonic crystals results from scattering and diffraction of light by a periodic structure, ${ }^{140,141}$ which can be observed in nature widely. To obtain SC of visible light, two factors should be followed: 
a)

a) $\quad 6.6 \mathrm{~nm}$
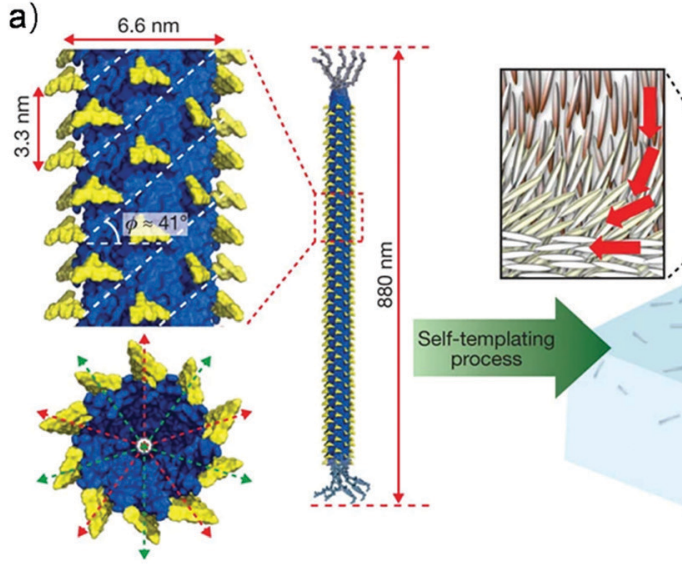

$\int_{\text {uulling }}$
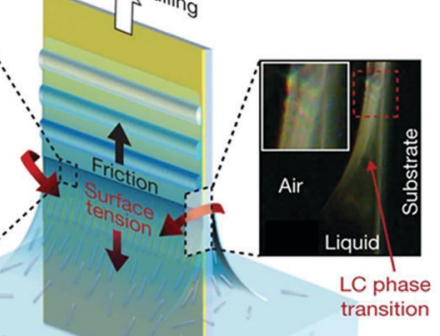

b)

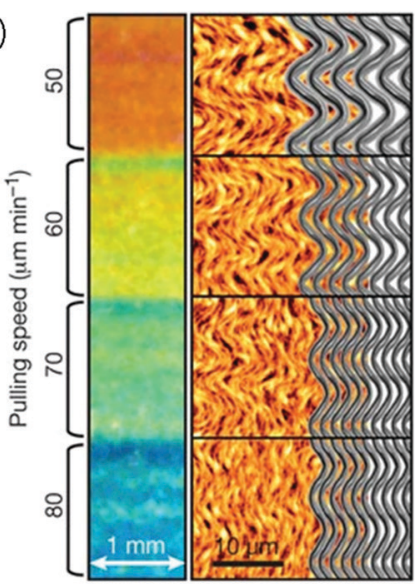

c)

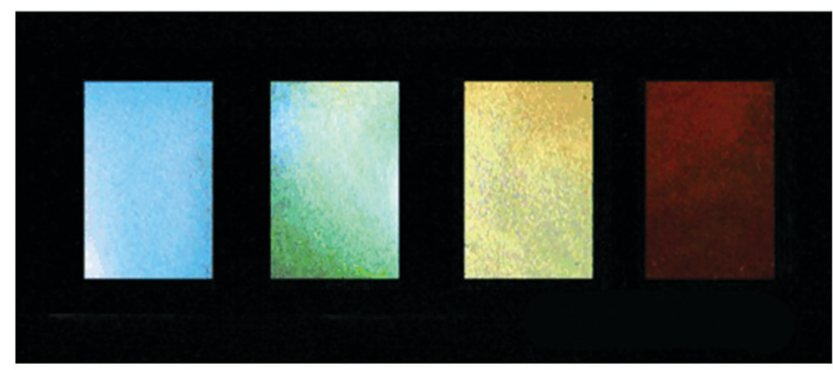

e)

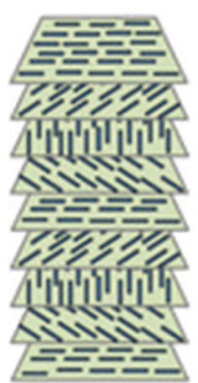

f)

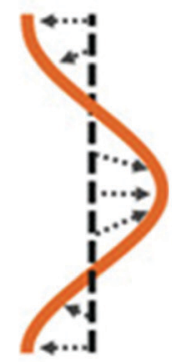

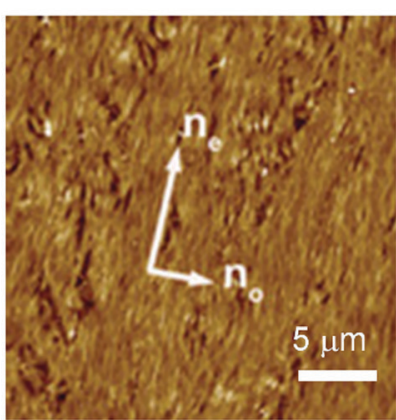

d)

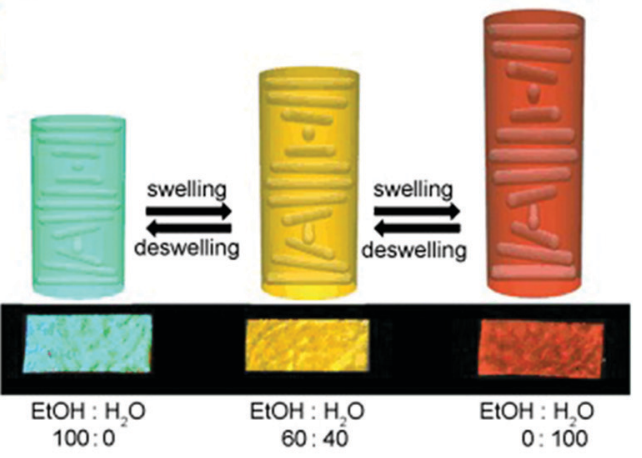

g)

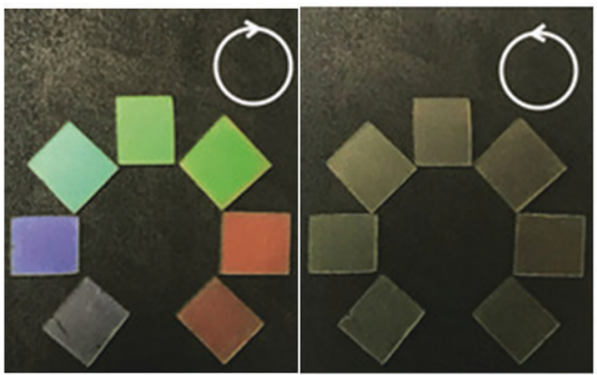

Fig. 13 Structural color induced by an ordered NW array. (a) Schematic of the self-templated assembly of chiral colloidal particles into structural color materials. (b) Different structural color resulted from various pulling speeds. (c) Increasing the ratio of the PF precursor to CNCs caused a red shift of colors (from right to left: CP1a, CP1b, CP1c, and CP1d). (d) The swelling behavior of the mesoporous plastics in mixtures of water and ethanol. (e) Schematic illustration of the chiral structure. (f) The image of the ordered NW array. (g) NW multilayer films viewed through a right- and left-polarizer. ( $a$ and b) Reprinted with permission from ref. 162. Copyright 2011 Nature Publishing Group. (c and d) Reprinted with permission from ref. 163. Copyright 2013 Wiley. (e-g) Reprinted with permission from ref. 159. Copyright 2019 Wiley.

(a) A periodic structure with a feature size comparable to the visible light wavelength is required.

(b) The dielectric material should have less absorption in the visible light wave range to reflect ample visible light.

1D nanomaterials can be assembled into an ordered structure for SC too. ${ }^{140,142,143}$ Firstly, NWs could be assembled with a layer-by-layer structure. An ultrathin NW monolayer provides the flexibility for adjusting the value of the periodic feature size, which dominates the reflection peak of SC. Besides, the anisotropic optical property of an aligned NW film induces a chiroptical effect with a right or left-circular polarization characteristic. For a typical NW chiral SC device, the NWs are aligned in uniform orientation in each layer (thickness of $D$ ). Between the adjacent layers, the orientation has a clockwise or anticlockwise rotation of a specified angle $(\Theta)$ to get right or left-circularly polarized light. The Bragg reflection peak $\left(\lambda_{0}\right)$ is dependent on the refractive index $(n)$, incident angle $(\alpha)$, and helical pitch length $(L)$ when the rotation reaches $360^{\circ}$. The formula of this relationship can be expressed as below:

$$
\lambda_{0}=\sin \alpha \times n L=\sin \alpha \times n D \times 360^{\circ} / \Theta
$$

As shown in Fig. 13a, Chung and co-workers took advantage of self-templated assembly to produce ordered films with hierarchical 
structure and helical twist, which are composed of a special bacterial virus named M13 phage. ${ }^{144}$ The structures of the films, including nematic orthogonal twists, cholesteric helical ribbons, and smectic helicoidal nanofilaments, were realized by tuning the paraments affecting the kinetics or thermodynamics such as the M13 concentration, dragging speed, etc. Meanwhile, the films possess characteristic SC due to this hierarchical structure achieved by the ordered assembly (Fig. 13b). The smectic helicoidal nanofilaments could serve as a template for biomineralization, which demonstrated that this assembly method has enormous potential in the fields of optics and biology.

Cellulose nanocrystals could be self-assembled quickly in solution, which can be used for SC design. Mark and coworkers reported an interesting mesoporous phenol-formaldehyde resin with chiral order produced by self-assembled cellulose nanocrystals (CNCs), which exhibited changeable SC with various volume expansion (Fig. 13c). ${ }^{145}$ In this process, the selfassembled CNCs as a template played a key role in the chiral structure in the PF resin, resulting in unique optical properties. The Bragg reflection peak could be adjusted by the ratio of PF precursor to CNCs or adding salts such as NaCl. Moreover, the PF resin immersed in solvents with different polarities generated a different degree of expansion, leading to a redshift of the optical reflection peak owing to the changed helical pitch (Fig. 13d). Therefore, this material can be used in sensor applications and data secrecy.

Furthermore, a variety of CNC composite films were prepared by Zhou and co-workers, which have the feature of large scale and flexibility and consistent structural color. ${ }^{146}$ A simple two-step method was proposed to make them. The CNCs selfassembled fast in water and were then cured with phenolformaldehyde resins to form a film. For this compound, the SC was realized by the CNCs with helical nematic pitch comparable to the wavelength of light and the PF was mixed for the enhancement of thermal and mechanical properties. Meanwhile, the helical nematic pitch of the film could be controlled by both initial concentration of CNCs and the ambient humidity, resulting in abundant colors.

The self-templating and self-assembly methods cannot control the direction of rotation to get the required chiroptical effect due to the low controllability. Developing bottom-up assembly of NW multilayer films for programmable structure control can create precise chiroptical behavior, such as the LS method (Fig. 13e). Recently, a bottom-up method to fabricate SC ordered films composed of ultrafine $\mathrm{NiMoO}_{4} \cdot x \mathrm{H}_{2} \mathrm{O}$ NWs was demonstrated by Tang and co-workers. ${ }^{147}$ As presented in Fig. 13f, the NWs showed uniform orientation in the monolayer. The NWs showed a right-handed rotation structure due to the layer-by-layer alignment with clockwise rotation. Instead, the film possessed ordered chiral structure controlled by the varied rotation angle of the substrate in the assembly process. The reflection peak could be easily controlled by the rotation angle of adjacent layers to obtain reflected light from violet to red. NW rotated with a clockwise or anticlockwise direction can obtain a right/left-circular polarizer for reflecting dextrorotatory or laevogyrate light specifically (Fig. 13g). The researchers utilized CD spectroscopy to accurately characterize the optical activities of the photonic crystal, and all samples show obvious negative CD peaks at wavelength positions.

\subsection{Assembly of NWs for structural materials}

1D nanomaterials have strong intrinsic mechanical strength. In the assembly of 1D nanomaterials, the interactions between nanomaterial units dominate the macroscopic mechanical strength, especially NW fibers. A dense and ordered structure of NW fibers enhances the interaction with a larger surface contact area. ${ }^{148-150}$ In contrast, with a poorly aligned structure, the small contact area of units induces poor interaction with lower strength.

Assembly can also influence the strength of 2D NW films or 3D NW blocks. ${ }^{151-153}$ Firstly, an ordered NW structure can also reduce the gap between NWs, which can enhance the interactions for stronger materials. Also, the ordered alignment brings an anisotropic effect of mechanical strength. In 2D films, the strength is heavily affected by the NW orientation. ${ }^{154,155}$ In $3 \mathrm{D}$ blocks, the strength is related to both the orientation and stacking mode. ${ }^{152,156}$

Kraus et al. reported that an ordered structure of NWs was beneficial for stronger fibers of Au NWs. ${ }^{157}$ The assembly process was similar to polymer solution spinning. Au NW solution was injected into a coagulation bath containing an antisolvent that decreased the solution solubility and caused precipitation of NWs. The small-angle X-ray scattering pattern showed that the fibers had more ordered structure with faster flow velocity. It was as expected that the fibers had the largest tensile strength with well-aligned structure.

1D nanomaterials could also work as fillers to enhance the mechanical strength of polymer films. This system could be treated as a reinforced concrete structure, in which the NWs act as rebars due to the superior intrinsic strength and the polymer serves as concrete. If the NWs are distributed disorderly in the matrix, the NW rebars cannot reinforce the polymer efficiently. Only with unified alignment like a steel framework can all the NWs bear destructive force simultaneously for the strongest materials. Yu group presented a new method to increase the mechanical strength of crosslinked liquid-crystalline polymers (CLCPs) by embedding aligned carbon nanotubes. ${ }^{158}$ The CLCP mesogens distributed homogeneously along the length direction of the CNTs. Polarized optical micrographs and absorption spectra also showed that CLCP-CNTs had an ordered structure with optical anisotropy. The aligned CNTs brought a huge enhancement of the mechanical properties with anisotropy. The CLCP/CNT composite film showed the largest tensile strength of 31.2 MPa along the orientation of the CNTs, while the films showed a strength of 6.4 MPa in the vertical direction. Notably, the ordered nanotubes increased the strength of the CLCP films two times compared to the pure CLCP films. Wang also demonstrated new insight about how the order intensity could influence the strength of CNT films heavily. They used mechanical rolling with various winding rates to fabricate CNT films with different alignment. With a higher winding rate, the 
a)

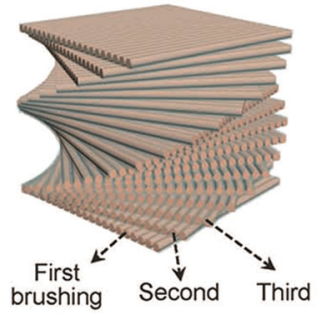

c)

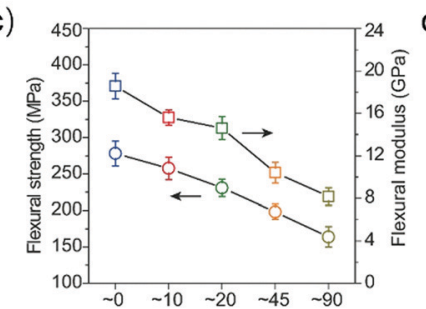

b)

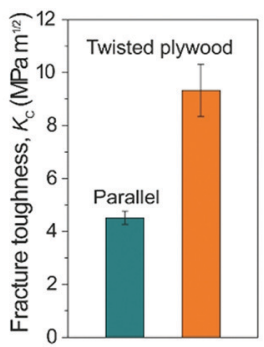

d)

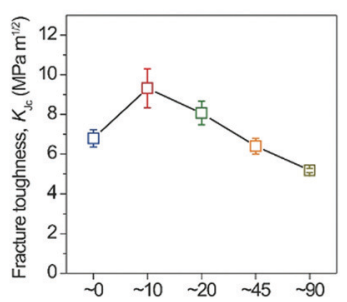

Fig. 14 The controllable mechanical strength of NW films by ordered structure. (a) Schematic illustration of twisted plywood structural materials. (b) Fracture toughness of 3D NW blocks with parallel and twisted assembly mode. The deviation angle influence on the flexural modulus (c) and fracture toughness (d). (a-d) Reprinted with permission from ref. 159. Copyright 2018 Science China Press.

CNTs became more well-aligned. The alignment level could also be reflected in the polarized Raman spectra due to the optical anisotropy of the ordered nanotube film. The assembly modulated the mechanical strength of the films effectively. The authors compared three samples: unaligned, slightly aligned, and well-aligned. The films showed a larger strength with the wellaligned structure. The well-aligned sample showed a strength of 2.8 GPa, which was 10 fold higher than the unaligned one.

The orientation of NWs in two adjacent layers influenced the strength of 3D NW LBL blocks. Our group demonstrated the

fabrication of macroscopic 2D artificial twisted films based on arranging 1D hydroxyapatite (HA) mineral microfibers by brushing-induced assembly methods. ${ }^{159}$ All the fibers showed the same orientation along the brush direction in one layer. For the next layer, the brush direction rotated by an angle to obtain a twisted structure (Fig. 14a). 3D bulk materials of the assembly could be fabricated by a laminating strategy by integrating the 2D films. As shown in Fig. 14b, the twisted structure showed two fold higher fracture toughness compared to the parallel samples. To explore the effect of alignment on the mechanical strength of the bulk, systematic mechanical tests were executed. As shown in Fig. 14c, with the deviation angle enlarged from $0^{\circ}$ to $90^{\circ}$, the flexural strength and modulus showed a decreasing trend. The toughness also changed with the angle. The $3 \mathrm{D}$ material with a $10^{\circ}-20^{\circ}$ deviation angle had the highest fracture toughness, which is two times higher than the $0^{\circ}$ deviation angle sample (Fig. 14d). The simulation data revealed that delamination, shear deformation, and rotation deformation occurred concurrently in the destructive process. When the deviation angle was small, delamination dominated the progress with high strength. With a large deviation angle, the shear and rotation effects dissipate more energy for a larger toughness. Through control of the alignment of fibers, we can obtain a plywood structural material with excellent mechanical performance.

\subsection{Assembly of NWs for energy applications}

In the energy field, mass transport plays an important role in improving the device capability. ${ }^{160,161}$ Ions and polar molecules prefer to move along larger electric field intensity regions induced by the electromagnetic force. 1D nanomaterials can be used for basic cells in a battery or a catalyst to accelerate ion migration. ${ }^{162-166} 1 \mathrm{D}$ nanomaterials form a locally enhanced a)

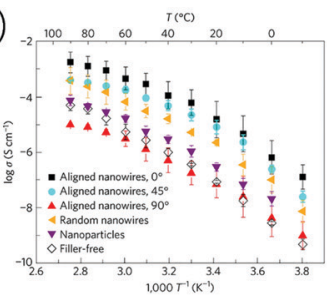

e)

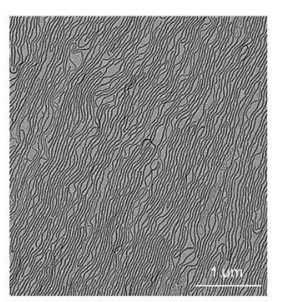

b)

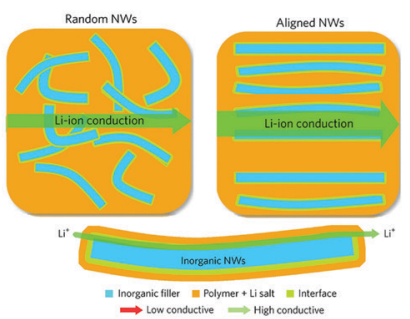

f)

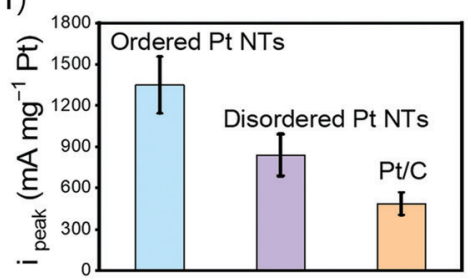

c)

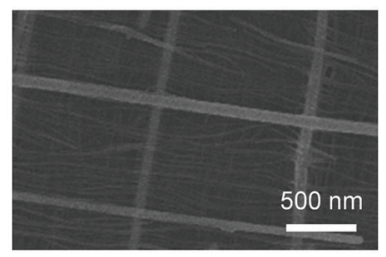

d)

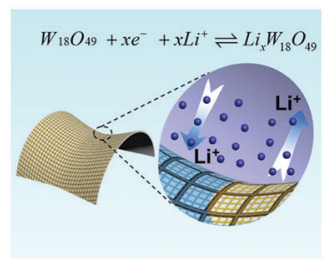

g)

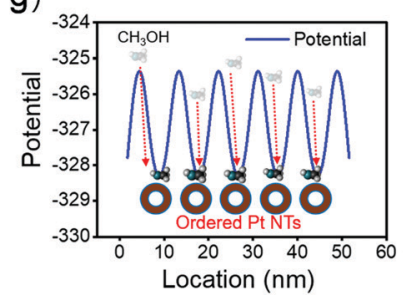

h)

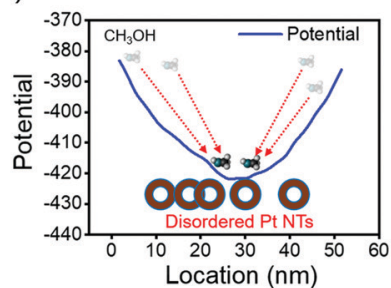

Fig. 15 Optimizing the kinetics of mass transport through ordered NW assembly. (a) Electrolyte conductivity of aligned NWs with different orientation and random architecture. (b) Schematic illustration of Li-ion conduction pathways in random and aligned NWs. (c) TEM of the co-assembly structure of the $\mathrm{Ag}-\mathrm{WO}_{x} \mathrm{NW}$ system. (d) The schematic of the optimized ion conduction induced by the ordered NW network. (e) TEM of large scale assembly of a Pt NT monolayer. (f) Histograms of $i_{\text {peak forward }}$ of Pt catalysts for the MOR. (g and h) The sectional potential of ordered (g) and random (h) NT film catalysts. ( $a$ and b) Reprinted with permission from ref. 167. Copyright 2017 Nature Publishing Group. (c and d) Reprinted with permission from ref. 168. Copyright 2017 American Chemical Society. (e-h) Reprinted with permission from ref. 170. Copyright 2019 American Chemical Society. 
Table 1 Summary of the improved performance of ordered NW assemblies

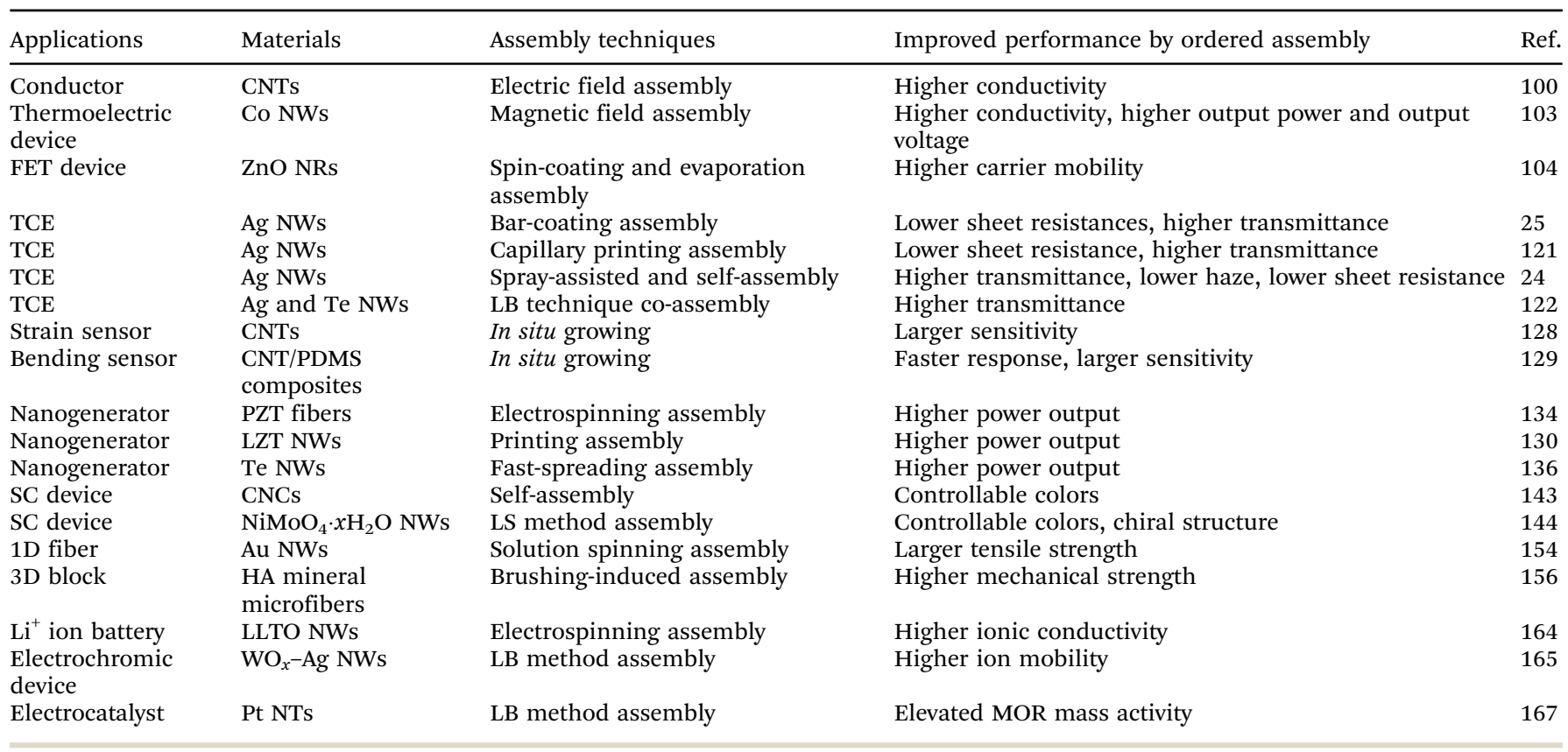

field around themselves in solution to guide migration of ions/ molecules. Different from a disordered structure, an ordered network forms a homogeneous electric field in a vast area. This ordered network avoids excessive ion/molecule aggregation in a small region in random samples to block matter transportation. This assembly strategy can be used in batteries, ionic electroactive polymers, and catalysts to accelerate the kinetics.

Cui et al. reported that aligned NWs as electrolyte fillers along the direction of electrodes could improve the ionic conductivity by one order of magnitude compared to the random sample. ${ }^{167}$ They fabricated well-ordered $\mathrm{Li}_{0.33} \mathrm{La}_{0.557}$ $\mathrm{TiO}_{3}$ (LLTO) NW arrays by electrospinning and cast PAN and $\mathrm{LiClO}_{4}$ solution on the fillers to construct a lithium-ion battery. The ionic conductivity of composite polymer electrolytes with aligned NWs could reach $6.05 \times 10^{-5} \mathrm{~S} \mathrm{~cm}^{-1}$ (Fig. 15a), which was 10 times high than random NW electrolytes $\left(5.40 \times 10^{-6} \mathrm{~S}\right.$ $\mathrm{cm}^{-1}$ ). The large conductivity enhancement was attributed to fast ion conduction on the NW surface in the axial direction without crossing junctions (Fig. 15b). Furthermore, the authors also demonstrated that the stability of electrolytes can be improved by assembly. The aligned NW array can also serve as an electrode of flexible lithium-ion batteries with high performance. Lu et al. prepared ultra-long, aligned carbon nanofiber thin films with embedded $\mathrm{In}_{2} \mathrm{O}_{3}$ nanocrystals for the anode of flexible batteries. The aligned nanofibers can act as a super-fast electron transfer channel and the regular space can promote diffusion of $\mathrm{Li}^{+}$ions.

The properties of NW-based electrochromic (EC) devices could be optimized by an ordered NW structure. ${ }^{168,169}$ EC devices composed of $\mathrm{WO}_{x} \mathrm{NWs}$ form dual-colors because of the intercalation and detachment of ions in $\mathrm{WO}_{x} \mathrm{NWs}$ induced by the electric field. Optimizing the migration of ions could improve the performance of EC devices. Our group reported using the co-assembly strategy to regulate the property of $\mathrm{WO}_{x}-\mathrm{Ag}$ NW-based EC films. ${ }^{168}$ As shown in Fig. 15c, Ag NWs served as conductive components and the $\mathrm{WO}_{x} \mathrm{NWs}$ played a role of spacers to separate $\mathrm{Ag}$ NWs and worked as EC media. Due to the uniform distribution of Ag NWs in the electrode, the homogeneous electric field will accelerate the migration of ions on a large scale resulting in a high EC performance (Fig. 15d). Also, when the ratio of $\mathrm{Ag}: \mathrm{WO}_{x} \mathrm{NWs}$ in the electrode increases, it could reduce the coloration/bleaching time due to the enhanced electric field.

The assembly of NWs can also be applied in the electrocatalytic field for optimized mass transfer. Our group reported a new strategy to optimize catalytic kinetics by well-designed ordered nanotube catalysts. ${ }^{170}$ As shown in Fig. 15e, Pt NTs were assembled by the LB technique to form an ordered structure for the methanol oxidation reaction, while the disordered sample by dip-coating worked as the control group. It was very impressive that the aligned sample showed an elevated MOR mass activity of $1349.4 \mathrm{~mA} \mathrm{mg}^{-1}$, which was 1.8-fold higher than the control disordered group (Fig. 15f). The mechanism of the order-enhanced catalytic performance was studied in detail. The potential of the aligned array showed a periodic Gaussian-like change as shown in Fig. 15g. So, the $\mathrm{CH}_{3} \mathrm{OH}$ molecules were well distributed on the surface of NWs induced by a periodic electric potential. This meant that all the nanotubes receive an equal number of molecules for catalysis. But when it came to a random sample, the potential seemed to be a large valley (Fig. 15h). All the $\mathrm{CH}_{3} \mathrm{OH}$ molecules flowed to the valley bottom under the inhomogeneous electric field. Only a small number of NTs were still working efficiently. Notably, this assembly induced electrocatalytic performance enhancement strategy could be extended to many NW catalyst systems. 


\section{Conclusions and outlook}

1D assembled nanomaterials have received an enormous amount of attention since increasing progress has been made in synthesis of nanomaterials, and thus the applications of NW devices have become a new emergent focus. In this review, we have overviewed the structure-property relationship of assembled NW materials and highlighted the latest advancements and examples emerging in this research area. Ordered NW structures with anisotropic properties have been summarized here, including anisotropic conduction, optical anisotropy, thermal anisotropy, magnetic anisotropy, and anisotropic strain sensors. The mechanism of this phenomenon is that $1 \mathrm{D}$ nanomaterials have plenty of transport channels, which can direct the conduction of electrons, phonons, and ions along their longitudinal direction, and could be used for an efficient transport medium, which means that altering the alignment could optimize the transport speed. Besides the structural features of assembled NW materials, it is necessary to realize the performance enhancement of ordered 1D nanomaterial-based devices to satisfy the needs of applications. As we know, 1D nanomaterial junctions play an important role in the nanomaterial network. For example, the resistance of a metal NW film is mainly dependent on the junction resistance which is much larger than the resitance of NW itself. Aligning the NWs with a same direction will reduce the junction resistance greatly, which improves the conductivity of the NW device. The space of the NW network could also be regulated by alignment. With precisely designed microstructure of assemblies, the interaction between 1D nanomaterials is changeable. Last, with advanced assembly methods, the configuration and function of a NW device can be predefined. For example, multiple NWs form ordered patterns on substrate to work as an integrated circuit. All the excellent performances induced by ordered assembly are summarized in Table 1. This unique superiority of ordered assembly brings a bright future to the commercial applications of NW based devices.

Although multitudinous benefits could be obtained from ordered NW assembly systems, there are a lot of problems and challenges to solve. In general, the first problem is that the origin of performance improvement by assembly configurations is still not clear in many NW devices. Although great achievements have been reported on NW alignment, the ordered assembly effect seems to be multiple. Establishing a physics or chemistry model to simulate devices' working processes can be an effective way to further understand the mechanism of performance enhancements. For example, electric field simulation with various intensity and frequencies could point out the optimized field parameters for aligning NWs with various architecture. This simulation can also be guidance for designing new high-performance NW devices. The contradiction between process cost and performance is another question that needs to be balanced urgently. Although ordered assembly can bring huge performance improvement from the structure design, the existing assembly techniques are costly and timeconsuming, which is not suitable for commercial production. How to develop a new technique enabled large scale and give continuous-production is the key to commercial applications of NW devices. In general, NW array is in short range ordering in the most previous reports on NW assemblies. A large scale ordered NW array is usually more difficult to prepare. In addition, the reported assembly methods are often manual for more elaborate control. To solve this problem, we should focus on NW assembly interfacial science, such as the airliquid interface of the LB technique, and the liquid-substrate interface of shear force assembly. Further understanding the dynamic and thermodynamic processes of assembly will allow it possible to optimize the assembly conditions for larger scale and fast assembly.

\section{Conflicts of interest}

There are no conflicts to declare.

\section{Acknowledgements}

We acknowledge the funding support from the National Natural Science Foundation of China (Grants 21922204, 21771168, 51732011, 21431006, 21761132008), the Foundation for Innovative Research Groups of the National Natural Science Foundation of China (Grant 21521001), Key Research Program of Frontier Sciences, CAS (Grant QYZDJ-SSW-SLH036), the National Basic Research Program of China (Grant 2014CB931800), the Users with Excellence and Scientific Research Grant of Hefei Science Center of CAS (2015HSC-UE007), the Fundamental Research Funds for the Central Universities (WK2100000005), and the Joint Funds from Hefei National Synchrotron Radiation Laboratory (UN2018LHJJ).

\section{Notes and references}

1 M. H. Huang, S. Mao, H. Feick, H. Yan, Y. Wu, H. Kind, E. Weber, R. Russo and P. Yang, Room-temperature ultraviolet nanowire nanolasers, Science, 2001, 292(5523), 1897-1899.

2 L. Hu, H. S. Kim, J. Y. Lee, P. Peumans and Y. Cui, Scalable coating and properties of transparent, flexible, silver nanowire electrodes, ACS Nano, 2010, 4(5), 2955-2963.

3 Y. Cui, Q. Wei, H. Park and C. M. Lieber, Nanowire nanosensors for highly sensitive and selective detection of biological and chemical species, Science, 2001, 293(5533), 1289-1292.

4 X. Wang, J. Song, J. Liu and Z. L. Wang, Direct-current nanogenerator driven by ultrasonic waves, Science, 2007, 316(5821), 102-105.

5 J. Mao, W. Chen, D. He, J. Wan, J. Pei, J. Dong, Y. Wang, P. An, Z. Jin, W. Xing, H. Tang, Z. Zhuang, X. Liang, Y. Huang, G. Zhou, L. Wang, D. Wang and Y. Li, Design of ultrathin Pt-Mo-Ni nanowire catalysts for ethanol electrooxidation, Sci. Adv., 2017, 3(8), e1603068.

6 R. X. Yan, D. Gargas and P. D. Yang, Nanowire photonics, Nat. Photonics, 2009, 3(10), 569-576. 
7 N. P. Dasgupta, J. Sun, C. Liu, S. Brittman, S. C. Andrews, J. Lim, H. Gao, R. Yan and P. Yang, 25th anniversary article: semiconductor nanowires-synthesis, characterization, and applications, Adv. Mater., 2014, 26(14), 2137-2184.

8 Z. Liu, J. Xu, D. Chen and G. Shen, Flexible electronics based on inorganic nanowires, Chem. Soc. Rev., 2015, 44(1), 161-192.

9 J. Deng, Y. Su, D. Liu, P. Yang, B. Liu and C. Liu, Nanowire Photoelectrochemistry, Chem. Rev., 2019, 119(15), 9221-9259.

10 L. N. Quan, J. Kang, C. Z. Ning and P. Yang, Nanowires for Photonics, Chem. Rev., 2019, 119(15), 9153-9169.

11 Y. N. Xia, P. D. Yang, Y. G. Sun, Y. Y. Wu, B. Mayers, B. Gates, Y. D. Yin, F. Kim and Y. Q. Yan, One-dimensional nanostructures: Synthesis, characterization, and applications, Adv. Mater., 2003, 15(5), 353-389.

12 Y. Zhao, S. S. You, A. Zhang, J. H. Lee, J. Huang and C. M. Lieber, Scalable ultrasmall three-dimensional nanowire transistor probes for intracellular recording, Nat. Nanotechnol., 2019, 14(8), 783-790.

13 J. T. Hu, T. W. Odom and C. M. Lieber, Chemistry and physics in one dimension: Synthesis and properties of nanowires and nanotubes, Acc. Chem. Res., 1999, 32(5), 435-445.

14 M. S. Gudiksen, L. J. Lauhon, J. Wang, D. C. Smith and C. M. Lieber, Growth of nanowire superlattice structures for nanoscale photonics and electronics, Nature, 2002, 415(6872), 617-620.

15 L. Guniat, P. Caroff and I. M. A. Fontcuberta, Vapor Phase Growth of Semiconductor Nanowires: Key Developments and Open Questions, Chem. Rev., 2019, 119(15), 8958-8971.

16 M. Yazawa, M. Koguchi, A. Muto, M. Ozawa and K. Hiruma, Effect of One Monolayer of Surface Gold Atoms on the Epitaxial-Growth of Inas Nanowhiskers, Appl. Phys. Lett., 1992, 61(17), 2051-2053.

17 G. Yu, J. Gao, J. C. Hummelen, F. Wudl and A. J. Heeger, Polymer Photovoltaic Cells - Enhanced Efficiencies Via a Network of Internal Donor-Acceptor Heterojunctions, Science, 1995, 270(5243), 1789-1791.

18 H. J. Dai, E. W. Wong, Y. Z. Lu, S. S. Fan and C. M. Lieber, Synthesis and Characterization of Carbide Nanorods, Nature, 1995, 375(6534), 769-772.

19 J. W. Liu, H. W. Liang and S. H. Yu, Macroscopic-scale assembled nanowire thin films and their functionalities, Chem. Rev., 2012, 112(8), 4770-4799.

20 S. G. Park, M. Kang, S. Kim, H. S. Jung and D. H. Kim, 3Dassembled $\mathrm{Ag}$ nanowires for use in plasmon-enhanced spectroscopic sensors, Appl. Spectrosc. Rev., 2019, 54(4), 325-347.

21 S. Jayadev, E. J. Steinbart, Y. Y. Chi, W. A. Kukull, G. D. Schellenberg and T. D. Bird, Conjugal Alzheimer disease: risk in children when both parents have Alzheimer disease, Arch. Neurol., 2008, 65(3), 373-378.

22 S. Zhao, F. Han, J. Li, X. Meng, W. Huang, D. Cao, G. Zhang, R. Sun and C. P. Wong, Advancements in Copper Nanowires: Synthesis, Purification, Assemblies, Surface Modification, and Applications, Small, 2018, 14(26), e1800047.
23 R. Bian, L. Meng, M. Zhang, L. Chen and H. Liu, Aligning One-Dimensional Nanomaterials by Solution Processes, ACS Omega, 2019, 4(1), 1816-1823.

24 J. Xiong, S. Li, Y. Ye, J. Wang, K. Qian, P. Cui, D. Gao, M. F. Lin, T. Chen and P. S. Lee, A Deformable and Highly Robust Ethyl Cellulose Transparent Conductor with a Scalable Silver Nanowires Bundle Micromesh, Adv. Mater., 2018, 30(36), e1802803.

25 S. Cho, S. Kang, A. Pandya, R. Shanker, Z. Khan, Y. Lee, J. Park, S. L. Craig and H. Ko, Large-Area Cross-Aligned Silver Nanowire Electrodes for Flexible, Transparent, and Force-Sensitive Mechanochromic Touch Screens, ACS Nano, 2017, 11(4), 4346-4357.

26 A. R. Tao, J. Huang and P. Yang, Langmuir-Blodgettry of nanocrystals and nanowires, Acc. Chem. Res., 2008, 41(12), 1662-1673.

27 J. W. Liu, J. H. Zhu, C. L. Zhang, H. W. Liang and S. H. Yu, Mesostructured assemblies of ultrathin superlong tellurium nanowires and their photoconductivity, J. Am. Chem. Soc., 2010, 132(26), 8945-8952.

28 W. R. Huang, Z. He, J. L. Wang, J. W. Liu and S. H. Yu, Mass Production of Nanowire-Nylon Flexible Transparent Smart Windows for PM2.5 Capture, iScience, 2019, 12, 333-341.

29 W.-b. Wei, K. Chen and G.-l. Ge, Electrostatically controlled nematic and smectic assembly of gold nanorods, Chem. Res. Chin. Univ., 2013, 29(5), 929-933.

30 L. Meng, R. Bian, C. Guo, B. Xu, H. Liu and L. Jiang, Aligning Ag Nanowires by a Facile Bioinspired Directional Liquid Transfer: Toward Anisotropic Flexible Conductive Electrodes, Adv. Mater., 2018, 30(25), e1706938.

31 K. M. Ryan, A. Mastroianni, K. A. Stancil, H. Liu and A. P. Alivisatos, Electric-field-assisted assembly of perpendicularly oriented nanorod superlattices, Nano Lett., 2006, 6(7), 1479-1482.

32 D. Fragouli, R. Buonsanti, G. Bertoni, C. Sangregorio, C. Innocenti, A. Falqui, D. Gatteschi, P. D. Cozzoli, A. Athanassiou and R. Cingolani, Dynamical formation of spatially localized arrays of aligned nanowires in plastic films with magnetic anisotropy, ACS Nano, 2010, 4(4), 1873-1878.

33 I. Hwang, M. Seong, H. Yi, H. Ko, H. H. Park, J. Yeo, W. G. Bae, H. W. Park and H. E. Jeong, Low-Resistant Electrical and Robust Mechanical Contacts of SelfAttachable Flexible Transparent Electrodes with Patternable Circuits, Adv. Funct. Mater., 2020, 30(17), 2000458.

34 J. Kim, D. Ouyang, H. F. Lu, F. Ye, Y. W. Guo, N. Zhao and W. C. H. Choy, High Performance Flexible Transparent Electrode via One-Step Multifunctional Treatment for Ag Nanonetwork Composites Semi-Embedded in LowTemperature-Processed Substrate for Highly Performed Organic Photovoltaics, Adv. Energy Mater., 2020, 10(15), 1903919.

35 G. Hills, C. Lau, A. Wright, S. Fuller, M. D. Bishop, T. Srimani, P. Kanhaiya, R. Ho, A. Amer, Y. Stein, D. Murphy, C. Arvind, A. Chandrakasan and M. M. Shulaker, Modern microprocessor 
built from complementary carbon nanotube transistors, Nature, 2019, 572(7771), 595-602.

36 H. Hu, S. Wang, X. Feng, M. Pauly, G. Decher and Y. Long, In-plane aligned assemblies of 1D-nanoobjects: recent approaches and applications, Chem. Soc. Rev., 2020, 49(2), 509-553.

37 Z. He, Y. Yang, H. W. Liang, J. W. Liu and S. H. Yu, Nanowire Genome: A Magic Toolbox for 1D Nanostructures, Adv. Mater., 2019, 31(51), e1902807.

38 S. C. Glotzer and M. J. Solomon, Anisotropy of building blocks and their assembly into complex structures, Nat. Mater., 2007, 6(8), 557-562.

39 C. Jia, Z. Lin, Y. Huang and X. Duan, Nanowire Electronics: From Nanoscale to Macroscale, Chem. Rev., 2019, 119(15), 9074-9135.

40 J. L. Wang, M. Hassan, J. W. Liu and S. H. Yu, Nanowire Assemblies for Flexible Electronic Devices: Recent Advances and Perspectives, Adv. Mater., 2018, 30(48), e1803430.

41 Y. Z. Long, M. Yu, B. Sun, C. Z. Gu and Z. Fan, Recent advances in large-scale assembly of semiconducting inorganic nanowires and nanofibers for electronics, sensors and photovoltaics, Chem. Soc. Rev., 2012, 41(12), 4560-4580.

42 B. Su, Y. Wu and L. Jiang, The art of aligning onedimensional (1D) nanostructures, Chem. Soc. Rev., 2012, 41(23), 7832-7856.

43 R. K. Joshi and J. J. Schneider, Assembly of one dimensional inorganic nanostructures into functional 2D and 3D architectures. Synthesis, arrangement and functionality, Chem. Soc. Rev., 2012, 41(15), 5285-5312.

44 L. Mai, X. Tian, X. Xu, L. Chang and L. Xu, Nanowire electrodes for electrochemical energy storage devices, Chem. Rev., 2014, 114(23), 11828-11862.

45 J. Ji, Z. Zhou, X. Yang, W. Zhang, S. Sang and P. Li, OneDimensional Nano-Interconnection Formation, Small, 2013, 9(18), 3014-3029.

46 R. K. Joshi and J. J. Schneider, Assembly of one dimensional inorganic nanostructures into functional 2D and 3D architectures. Synthesis, arrangement and functionality, Chem. Soc. Rev., 2012, 41(15), 5285-5312.

47 W. Li, Q. Yue, Y. Deng and D. Zhao, Ordered Mesoporous Materials Based on Interfacial Assembly and Engineering, Adv. Mater., 2013, 25(37), 5129-5152.

48 B. Su, Y. Wu and L. Jiang, The art of aligning one-dimensional (1D) nanostructures, Chem. Soc. Rev., 2012, 41(23), 7832-7856.

49 W. Zhang and S. Yang, In Situ Fabrication of Inorganic Nanowire Arrays Grown from and Aligned on Metal Substrates, Acc. Chem. Res., 2009, 42(10), 1617-1627.

50 R. Zhang, Y. Zhang and F. Wei, Horizontally aligned carbon nanotube arrays: growth mechanism, controlled synthesis, characterization, properties and applications, Chem. Soc. Rev., 2017, 46(12), 3661-3715.

51 D. Ye, Y. Ding, Y. Duan, J. Su, Z. Yin and Y. A. Huang, Large-Scale Direct-Writing of Aligned Nanofibers for Flexible Electronics, Small, 2018, 14(21), 1703521.

52 S. Zhao, F. Han, J. Li, X. Meng, W. Huang, D. Cao, G. Zhang, R. Sun and C.-P. Wong, Advancements in Copper Nanowires:
Synthesis, Purification, Assemblies, Surface Modification, and Applications, Small, 2018, 14(26), 1800047.

53 Y. Xu, X. B. Chen, B. L. Gu and W. H. Duan, Intrinsic anisotropy of thermal conductance in graphene nanoribbons, Appl. Phys. Lett., 2009, 95(23), 233116.

54 J. Elser, R. Wangberg, V. A. Podolskiy and E. E. Narimanov, Nanowire metamaterials with extreme optical anisotropy, Appl. Phys. Lett., 2006, 89(26), 261102.

55 G. R. Patzke, F. Krumeich and R. Nesper, Oxidic nanotubes and nanorods-anisotropic modules for a future nanotechnology, Angew. Chem., Int. Ed., 2002, 41(14), 2446-2461.

56 J. Huang, R. Fan, S. Connor and P. Yang, One-step patterning of aligned nanowire arrays by programmed dip coating, Angew. Chem., Int. Ed., 2007, 46(14), 2414-2417.

57 B. Li, C. Zhang, B. Jiang, W. Han and Z. Lin, Flow-enabled self-assembly of large-scale aligned nanowires, Angew. Chem., Int. Ed., 2015, 54(14), 4250-4254.

58 G. Jo, J. W. Jeong, S. Choi, H. Kim, J. J. Park, J. Jung and M. Chang, Large-Scale Alignment of Polymer Semiconductor Nanowires for Efficient Charge Transport via Controlled Evaporation of Confined Fluids, ACS Appl. Mater. Interfaces, 2019, 11(1), 1135-1142.

59 X. Qi, Z. Lu, E. M. You, Y. He, Q. E. Zhang, H. J. Yi, D. Li, S. Y. Ding, Y. Jiang, X. Xiong, J. Xu, D. Ge, X. Y. Liu and H. Bai, Nanocombing Effect Leads to Nanowire-Based, inPlane, Uniaxial Thin Films, ACS Nano, 2018, 12(12), 12701-12712.

60 D. Kim, Y. K. Kim, S. C. Park, J. S. Ha, J. Huh, J. Na and G. T. Kim, Photoconductance of aligned $\mathrm{SnO} 2$ nanowire field effect transistors, Appl. Phys. Lett., 2009, 95(4), 043107.

61 A. Persano, M. De Giorgi, A. Fiore, R. Cingolani, L. Manna, A. Cola and R. Krahne, Photoconduction properties in aligned assemblies of colloidal CdSe/CdS nanorods, ACS Nano, 2010, 4(3), 1646-1652.

62 L. F. Shen, E. Y. B. Pun and J. C. Ho, Recent developments in III-V semiconducting nanowires for high-performance photodetectors, Mater. Chem. Front., 2017, 1(4), 630-645.

63 M. G. Zhu, J. Si, Z. Zhang and L. M. Peng, Aligning Solution-Derived Carbon Nanotube Film with Full Surface Coverage for High-Performance Electronics Applications, Adv. Mater., 2018, 30(23), e1707068.

64 S. Shi, L. D. Sun, Y. X. Xue, H. Dong, K. Wu, S. C. Guo, B. T. Wu and C. H. Yan, Scalable Direct Writing of Lanthanide-Doped KMnF3 Perovskite Nanowires into Aligned Arrays with Polarized Up-Conversion Emission, Nano Lett., 2018, 18(5), 2964-2969.

$65 \mathrm{~J} . \mathrm{Hu}, \mathrm{L}$. Li, W. Yang, L. Manna, L. Wang and A. P. Alivisatos, Linearly polarized emission from colloidal semiconductor quantum rods, Science, 2001, 292(5524), 2060-2063.

66 Y. Che, Y. Wang, T. You, H. Chang, P. Yin and J. Zhai, Multispectral Plasmon of Anisotropic Core-shell Gold Nanorods@SiO2: Dual-band Absorption Enhancement with Coupling Dye Molecules, Chem. Res. Chin. Univ., 2018, 34(5), 772-780. 
67 J. J. Cheng, E. H. Hill, Y. B. Zheng, T. C. He and Y. J. Liu, Optically active plasmonic resonance in self-assembled nanostructures, Mater. Chem. Front., 2018, 2(4), 662-678.

68 S. J. Boehm, L. Kang, D. H. Werner and C. D. Keating, Field-Switchable Broadband Polarizer Based on Reconfigurable Nanowire Assemblies, Adv. Funct. Mater., 2017, 27(5), 1604703.

69 S. Zhang, W. Shi, T. D. Siegler, X. Gao, F. Ge, B. A. Korgel, Y. He, S. Li and X. Wang, An All-Inorganic Colloidal Nanocrystal Flexible Polarizer, Angew. Chem., Int. Ed., 2019, 58(26), 8730-8735.

70 T. Du, J. Schneider, A. K. Srivastava, A. S. Susha, V. G. Chigrinov, H. S. Kwok and A. L. Rogach, Combination of Photoinduced Alignment and Self-Assembly to Realize Polarized Emission from Ordered Semiconductor Nanorods, ACS Nano, 2015, 9(11), 11049-11055.

71 M. Hasegawa, Y. Hirayama and S. Dertinger, Polarized fluorescent emission from aligned electrospun nanofiber sheets containing semiconductor nanorods, Appl. Phys. Lett., 2015, 106(5), 051103.

72 N. Zhou, Y. Bekenstein, C. N. Eisler, D. Zhang, A. M. Schwartzberg, P. Yang, A. P. Alivisatos and J. A. Lewis, Perovskite nanowire-block copolymer composites with digitally programmable polarization anisotropy, Sci. Adv., 2019, 5(5), eaav8141.

73 A. K. Menon, I. Haechler, S. Kaur, S. Lubner and R. S. Prasher, Enhanced solar evaporation using a photothermal umbrella for wastewater management, Nat. Sustain., 2020, 3(2), 144-151.

74 Y. Xu, D. Kraemer, B. Song, Z. Jiang, J. Zhou, J. Loomis, J. Wang, M. Li, H. Ghasemi, X. Huang, X. Li and G. Chen, Nanostructured polymer films with metal-like thermal conductivity, Nat. Commun., 2019, 10(1), 1771.

75 E. S. Choi, J. S. Brooks, D. L. Eaton, M. S. Al-Haik, M. Y. Hussaini, H. Garmestani, D. Li and K. Dahmen, Enhancement of thermal and electrical properties of carbon nanotube polymer composites by magnetic field processing, J. Appl. Phys., 2003, 94(9), 6034-6039.

76 T. Terao, C. Y. Zhi, Y. Bando, M. Mitome, C. C. Tang and D. Golberg, Alignment of Boron Nitride Nanotubes in Polymeric Composite Films for Thermal Conductivity Improvement, J. Phys. Chem. C, 2010, 114(10), 4340-4344.

77 H. Huang, C. H. Liu, Y. Wu and S. S. Fan, Aligned carbon nanotube composite films for thermal management, $A d v$. Mater., 2005, 17(13), 1652-1656.

78 F. Deng, Q. S. Zheng, L. F. Wang and C. W. Nan, Effects of anisotropy, aspect ratio, and nonstraightness of carbon nanotubes on thermal conductivity of carbon nanotube composites, Appl. Phys. Lett., 2007, 90(2), 021914.

79 Y. C. Lan, Y. Wang and Z. F. Ren, Physics and applications of aligned carbon nanotubes, Adv. Phys., 2011, 60(4), 553-678.

80 Z. M. Shen and J. C. Feng, Achieving vertically aligned SiC microwires networks in a uniform cold environment for polymer composites with high through-plane thermal conductivity enhancement, Compos. Sci. Technol., 2019, 170, 135-140.
81 Y. Yao, X. Zhu, X. Zeng, R. Sun, J. B. Xu and C. P. Wong, Vertically Aligned and Interconnected SiC Nanowire Networks Leading to Significantly Enhanced Thermal Conductivity of Polymer Composites, ACS Appl. Mater. Interfaces, 2018, 10(11), 9669-9678.

82 A. M. Marconnet, N. Yamamoto, M. A. Panzer, B. L. Wardle and K. E. Goodson, Thermal conduction in aligned carbon nanotube-polymer nanocomposites with high packing density, ACS Nano, 2011, 5(6), 4818-4825.

83 D. Fragouli, R. Buonsanti, G. Bertoni, C. Sangregorio, C. Innocenti, A. Falqui, D. Gatteschi, P. D. Cozzoli, A. Athanassiou and R. Cingolani, Dynamical Formation of Spatially Localized Arrays of Aligned Nanowires in Plastic Films with Magnetic Anisotropy, ACS Nano, 2010, 4(4), 1873-1878.

84 M. Hassan, H. J. Zhan, J. L. Wang, J. W. Liu and J. F. Chen, Self-Assembly Anisotropic Magnetic Nanowire Films Induced by External Magnetic Field, ChemistryOpen, 2020, 9(5), 588-592.

85 D. Fragouli, A. Das, C. Innocenti, Y. Guttikonda, S. Rahman, L. Liu, V. Caramia, C. M. Megaridis and A. Athanassiou, Polymeric films with electric and magnetic anisotropy due to magnetically assembled functional nanofibers, ACS Appl. Mater. Interfaces, 2014, 6(6), 4535-4541.

86 S. Hong, J. Lee, K. Do, M. Lee, J. H. Kim, S. Lee and D. H. Kim, Stretchable Electrode Based on Laterally Combed Carbon Nanotubes for Wearable Energy Harvesting and Storage Devices, Adv. Funct. Mater., 2017, 27(48), 1704353.

87 L. Li, Y. Bai, L. Li, S. Wang and T. Zhang, A Superhydrophobic Smart Coating for Flexible and Wearable Sensing Electronics, Adv. Mater., 2017, 29(43), 1702517.

88 Y. J. Fan, X. Li, S. Y. Kuang, L. Zhang, Y. H. Chen, L. Liu, K. Zhang, S. W. Ma, F. Liang, T. Wu, Z. L. Wang and G. Zhu, Highly Robust, Transparent, and Breathable Epidermal Electrode, ACS Nano, 2018, 12(9), 9326-9332.

89 S. Chen, Y. J. Song, D. Y. Ding, Z. Ling and F. Xu, Flexible and Anisotropic Strain Sensor Based on Carbonized Crepe Paper with Aligned Cellulose Fibers, Adv. Funct. Mater., 2018, 28(42), 1802547.

90 J. H. Lee, J. Kim, D. Liu, F. Guo, X. Shen, Q. Zheng, S. Jeon and J. K. Kim, Highly Aligned, Anisotropic Carbon Nanofiber Films for Multidirectional Strain Sensors with Exceptional Selectivity, Adv. Funct. Mater., 2019, 29(29), 1901623.

91 H. H. Chou, A. Nguyen, A. Chortos, J. W. To, C. Lu, J. Mei, T. Kurosawa, W. G. Bae, J. B. Tok and Z. Bao, A chameleoninspired stretchable electronic skin with interactive colour changing controlled by tactile sensing, Nat. Commun., 2015, 6, 8011.

92 X. Han, W. Du, M. Chen, X. Wang, X. Zhang, X. Li, J. Li, Z. Peng, C. Pan and Z. L. Wang, Visualization Recording and Storage of Pressure Distribution through a Smart Matrix Based on the Piezotronic Effect, Adv. Mater., 2017, 29(26), 1701253. 
93 M. Raisch, D. Genovese, N. Zaccheroni, S. B. Schmidt, M. L. Focarete, M. Sommer and C. Gualandi, Highly Sensitive, Anisotropic, and Reversible Stress/StrainSensors from Mechanochromic Nanofiber Composites, Adv. Mater., 2018, 30(39), e1802813.

94 S. Jiang, P. X. Hou, M. L. Chen, B. W. Wang, D. M. Sun, D. M. Tang, Q. Jin, Q. X. Guo, D. D. Zhang, J. H. Du, K. P. Tai, J. Tan, E. I. Kauppinen, C. Liu and H. M. Cheng, Ultrahigh-performance transparent conductive films of carbon-welded isolated single-wall carbon nanotubes, Sci. $A d v ., 2018,4(5)$, eaap9264.

$95 \mathrm{~F}$. Xu and Y. Zhu, Highly conductive and stretchable silver nanowire conductors, Adv. Mater., 2012, 24(37), 5117-5122.

96 S. Ye, A. R. Rathmell, Z. Chen, I. E. Stewart and B. J. Wiley, Metal nanowire networks: the next generation of transparent conductors, Adv. Mater., 2014, 26(39), 6670-6687.

97 X. Xiao, C. Lü, G. Wang, Y. Xu, J. Wang and H. Yang, Flexible triboelectric nanogenerator from micro-nano structured polydimethylsiloxane, Chem. Res. Chin. Univ., 2015, 31(3), 434-438.

98 J. Zhao, Inspired by Grape Seed and Wine: Tannic Acid as a Modified Coating for Fabricating Highly Flexible, Transparent and Conductive Film, Chem. Res. Chin. Univ., 2019, 35(5), 945-950.

99 H. B. Hu, S. C. Wang, S. C. Wang, G. W. Liu, T. Cao and Y. Long, Aligned Silver Nanowires Enabled Highly Stretchable and Transparent Electrodes with Unusual Conductive Property, Adv. Funct. Mater., 2019, 29(33), 1902922.

100 S. Thiemann, M. Gruber, I. Lokteva, J. Hirschmann, M. Halik and J. Zaumseil, High-mobility ZnO nanorod field-effect transistors by self-alignment and electrolytegating, ACS Appl. Mater. Interfaces, 2013, 5(5), 1656-1662.

101 B. R. Yang, W. Cao, G. S. Liu, H. J. Chen, Y. Y. Noh, T. Minari, H. C. Hsiao, C. Y. Lee, H. P. Shieh and C. Liu, Microchannel Wetting for Controllable Patterning and Alignment of Silver Nanowire with High Resolution, ACS Appl. Mater. Interfaces, 2015, 7(38), 21433-21441.

102 J. Shi, L. Liu, F. Xu and Y. Zhang, Improving the Photoresponse Properties of CdSe Quantum Wires by Alignment and Ligand Exchange, ACS Appl. Mater. Interfaces, 2019, 11(1), 1192-1200.

103 J. Ramon-Azcon, S. Ahadian, M. Estili, X. Liang, S. Ostrovidov, H. Kaji, H. Shiku, M. Ramalingam, K. Nakajima, Y. Sakka, A. Khademhosseini and T. Matsue, Dielectrophoretically aligned carbon nanotubes to control electrical and mechanical properties of hydrogels to fabricate contractile muscle myofibers, Adv. Mater., 2013, 25(29), 4028-4034.

104 Y. Ding, Y. Qiu, K. Cai, Q. Yao, S. Chen, L. Chen and J. He, High performance n-type Ag2Se film on nylon membrane for flexible thermoelectric power generator, Nat. Commun., 2019, 10(1), 841.

105 W. Zhou, Q. Fan, Q. Zhang, L. Cai, K. Li, X. Gu, F. Yang, N. Zhang, Y. Wang, H. Liu, W. Zhou and S. Xie, Highperformance and compact-designed flexible thermoelectric modules enabled by a reticulate carbon nanotube architecture, Nat. Commun., 2017, 8, 14886.
106 Y. N. Chen, M. H. He, J. H. Tang, G. C. Bazan and Z. Q. Liang, Flexible Thermoelectric Generators with Ultrahigh Output Power Enabled by Magnetic Field-Aligned Metallic Nanowires, Adv. Electron. Mater., 2018, 4(9), 1800200.

107 B. Sun and H. Sirringhaus, Surface tension and fluid flow driven self-assembly of ordered $\mathrm{ZnO}$ nanorod films for high-performance field effect transistors, J. Am. Chem. Soc., 2006, 128(50), 16231-16237.

108 J. Yao, H. Yan and C. M. Lieber, A nanoscale combing technique for the large-scale assembly of highly aligned nanowires, Nat. Nanotechnol., 2013, 8(5), 329-335.

109 I. Sanchez Esqueda, X. Yan, C. Rutherglen, A. Kane, T. Cain, P. Marsh, Q. Liu, K. Galatsis, H. Wang and C. Zhou, Aligned Carbon Nanotube Synaptic Transistors for Large-Scale Neuromorphic Computing, ACS Nano, 2018, 12(7), 7352-7361.

110 Z. Fan, J. C. Ho, Z. A. Jacobson, H. Razavi and A. Javey, Large-scale, heterogeneous integration of nanowire arrays for image sensor circuitry, Proc. Natl. Acad. Sci. U. S. A., 2008, 105(32), 11066-11070.

111 S. J. Kang, C. Kocabas, T. Ozel, M. Shim, N. Pimparkar, M. A. Alam, S. V. Rotkin and J. A. Rogers, Highperformance electronics using dense, perfectly aligned arrays of single-walled carbon nanotubes, Nat. Nanotechnol., 2007, 2(4), 230-236.

112 S. Y. Min, T. S. Kim, B. J. Kim, H. Cho, Y. Y. Noh, H. Yang, J. H. Cho and T. W. Lee, Large-scale organic nanowire lithography and electronics, Nat. Commun., 2013, 4, 1773.

113 L. Liu, J. Han, L. Xu, J. Zhou, C. Zhao, S. Ding, H. Shi, M. Xiao, L. Ding, Z. Ma, C. Jin, Z. Zhang and L. M. Peng, Aligned, high-density semiconducting carbon nanotube arrays for high-performance electronics, Science, 2020, 368(6493), 850-856.

$114 \mathrm{~J}$. Yao, H. Yan and C. M. Lieber, A nanoscale combing technique for the large-scale assembly of highly aligned nanowires, Nat. Nanotechnol., 2013, 8(5), 329-335.

115 H. Yan, H. S. Choe, S. Nam, Y. Hu, S. Das, J. F. Klemic, J. C. Ellenbogen and C. M. Lieber, Programmable nanowire circuits for nanoprocessors, Nature, 2011, 470(7333), 240-244.

116 Q. Cao, S. J. Han, G. S. Tulevski, Y. Zhu, D. D. Lu and W. Haensch, Arrays of single-walled carbon nanotubes with full surface coverage for high-performance electronics, Nat. Nanotechnol., 2013, 8(3), 180-186.

117 L. Kong, X. Zhang, Q. Tao, M. Zhang, W. Dang, Z. Li, L. Feng, L. Liao, X. Duan and Y. Liu, Doping-free complementary WSe2 circuit via van der Waals metal integration, Nat. Commun., 2020, 11(1), 1866.

118 S. Nam, X. Jiang, Q. Xiong, D. Ham and C. M. Lieber, Vertically integrated, three-dimensional nanowire complementary metal-oxide-semiconductor circuits, Proc. Natl. Acad. Sci. U. S. A., 2009, 106(50), 21035-21038.

119 K. Takei, T. Takahashi, J. C. Ho, H. Ko, A. G. Gillies, P. W. Leu, R. S. Fearing and A. Javey, Nanowire activematrix circuitry for low-voltage macroscale artificial skin, Nat. Mater., 2010, 9(10), 821-826. 
120 Y. Ge, J. Liu, X. Liu, J. Hu, X. Duan and X. Duan, Rapid Electrochemical Cleaning Silver Nanowire Thin Films for High-Performance Transparent Conductors, J. Am. Chem. Soc., 2019, 141(31), 12251-12257.

121 Z. Niu, F. Cui, Y. Yu, N. Becknell, Y. Sun, G. Khanarian, D. Kim, L. Dou, A. Dehestani, K. Schierle-Arndt and P. Yang, Ultrathin Epitaxial Cu@Au Core-Shell Nanowires for Stable Transparent Conductors, J. Am. Chem. Soc., 2017, 139(21), 7348-7354.

122 Z. Wu, Z. Chen, X. Du, J. M. Logan, J. Sippel, M. Nikolou, K. Kamaras, J. R. Reynolds, D. B. Tanner, A. F. Hebard and A. G. Rinzler, Transparent, conductive carbon nanotube films, Science, 2004, 305(5688), 1273-1276.

123 D. S. Hecht, L. Hu and G. Irvin, Emerging transparent electrodes based on thin films of carbon nanotubes, graphene, and metallic nanostructures, Adv. Mater., 2011, 23(13), 1482-1513.

124 S. Kang, T. Kim, S. Cho, Y. Lee, A. Choe, B. Walker, S. J. Ko, J. Y. Kim and H. Ko, Capillary Printing of Highly Aligned Silver Nanowire Transparent Electrodes for HighPerformance Optoelectronic Devices, Nano Lett., 2015, 15(12), 7933-7942.

125 J. W. Liu, J. L. Wang, Z. H. Wang, W. R. Huang and S. H. Yu, Manipulating nanowire assembly for flexible transparent electrodes, Angew. Chem., Int. Ed., 2014, 53(49), 13477-13482.

126 Y. Yang, J. L. Wang, L. Liu, Z. H. Wang, J. W. Liu and S. H. Yu, A room-temperature environmentally friendly solution process to assemble silver nanowire architectures for flexible transparent electrodes, Nanoscale, 2017, 9(1), 52-55.

127 L. Pan, G. Liu, W. Shi, J. Shang, W. R. Leow, Y. Liu, Y. Jiang, S. Li, X. Chen and R. W. Li, Mechano-regulated metalorganic framework nanofilm for ultrasensitive and antijamming strain sensing, Nat. Commun., 2018, 9(1), 3813.

128 F. Guo, Y. Jiang, Z. Xu, Y. Xiao, B. Fang, Y. Liu, W. Gao, P. Zhao, H. Wang and C. Gao, Highly stretchable carbon aerogels, Nat. Commun., 2018, 9(1), 881.

129 M. Amjadi, A. Pichitpajongkit, S. Lee, S. Ryu and I. Park, Highly stretchable and sensitive strain sensor based on silver nanowire-elastomer nanocomposite, ACS Nano, 2014, 8(5), 5154-5163.

130 K. K. Kim, S. Hong, H. M. Cho, J. Lee, Y. D. Suh, J. Ham and S. H. Ko, Highly Sensitive and Stretchable Multidimensional Strain Sensor with Prestrained Anisotropic Metal Nanowire Percolation Networks, Nano Lett., 2015, 15(8), 5240-5247.

131 T. Yamada, Y. Hayamizu, Y. Yamamoto, Y. Yomogida, A. Izadi-Najafabadi, D. N. Futaba and K. Hata, A stretchable carbon nanotube strain sensor for human-motion detection, Nat. Nanotechnol., 2011, 6(5), 296-301.

132 H. F. Zhu, X. W. Wang, J. Liang, H. L. Lv, H. Y. Tong, L. B. Ma, Y. Hu, G. Y. Zhu, T. Zhang, Z. X. Tie, Z. Liu, Q. W. Li, L. W. Chen, J. Liu and Z. Jin, Versatile Electronic Skins for Motion Detection of Joints Enabled by Aligned Few-Walled Carbon Nanotubes in Flexible Polymer Composites, Adv. Funct. Mater., 2017, 27(21), 1606604.
133 H. X. Tang, Y. R. Lin and H. A. Sodano, Enhanced Energy Storage in Nanocomposite Capacitors through Aligned PZT Nanowires by Uniaxial Strain Assembly, Adv. Energy Mater., 2012, 2(4), 469-476.

134 M. H. Malakooti, F. Jule and H. A. Sodano, Printed Nanocomposite Energy Harvesters with Controlled Alignment of Barium Titanate Nanowires, ACS Appl. Mater. Interfaces, 2018, 10(44), 38359-38367.

135 L. Persano, C. Dagdeviren, Y. Su, Y. Zhang, S. Girardo, D. Pisignano, Y. Huang and J. A. Rogers, High performance piezoelectric devices based on aligned arrays of nanofibers of poly(vinylidenefluoride-co-trifluoroethylene), Nat. Commun., 2013, 4, 1633.

136 J. Yan and Y. G. Jeong, High Performance Flexible Piezoelectric Nanogenerators based on BaTiO3 Nanofibers in Different Alignment Modes, ACS Appl. Mater. Interfaces, 2016, 8(24), 15700-15709.

137 H. Lee, H. Kim, D. Y. Kim and Y. Seo, Pure Piezoelectricity Generation by a Flexible Nanogenerator Based on Lead Zirconate Titanate Nanofibers, ACS Omega, 2019, 4(2), 2610-2617.

138 G. Zhu, R. Yang, S. Wang and Z. L. Wang, Flexible highoutput nanogenerator based on lateral $\mathrm{ZnO}$ nanowire array, Nano Lett., 2010, 10(8), 3151-3155.

139 T. I. Lee, S. Lee, E. Lee, S. Sohn, Y. Lee, S. Lee, G. Moon, D. Kim, Y. S. Kim, J. M. Myoung and Z. L. Wang, Highpower density piezoelectric energy harvesting using radially strained ultrathin trigonal tellurium nanowire assembly, Adv. Mater., 2013, 25(21), 2920-2925.

140 S. Biswas, D. Nepal, K. Park and R. A. Vaia, Orientation Sensing with Color Using Plasmonic Gold Nanorods and Assemblies, J. Phys. Chem. Lett., 2012, 3(18), 2568-2574.

141 R. M. Parker, G. Guidetti, C. A. Williams, T. Zhao, A. Narkevicius, S. Vignolini and B. Frka-Petesic, The SelfAssembly of Cellulose Nanocrystals: Hierarchical Design of Visual Appearance, Adv. Mater., 2018, 30(19), e1704477.

142 J. Lv, K. Hou, D. Ding, D. Wang, B. Han, X. Gao, M. Zhao, L. Shi, J. Guo, Y. Zheng, X. Zhang, C. Lu, L. Huang, W. Huang and Z. Tang, Gold Nanowire Chiral Ultrathin Films with Ultrastrong and Broadband Optical Activity, Angew. Chem., Int. Ed., 2017, 56(18), 5055-5060.

143 Z. Cheng, Y. Ma, L. Yang, F. Cheng, Z. J. Huang, A. Natan, H. Y. Li, Y. Chen, D. X. Cao, Z. F. Huang, Y. H. Wang, Y. M. Liu, R. D. Yang and H. L. Zhu, Plasmonic-Enhanced Cholesteric Films: Coassembling Anisotropic Gold Nanorods with Cellulose Nanocrystals, Adv. Opt. Mater., 2019, 7(9), 1801816.

144 W. J. Chung, J. W. Oh, K. Kwak, B. Y. Lee, J. Meyer, E. Wang, A. Hexemer and S. W. Lee, Biomimetic self-templating supramolecular structures, Nature, 2011, 478(7369), 364-368.

145 M. K. Khan, M. Giese, M. Yu, J. A. Kelly, W. Y. Hamad and M. J. MacLachlan, Flexible mesoporous photonic resins with tunable chiral nematic structures, Angew. Chem., Int. Ed., 2013, 52(34), 8921-8924.

146 K. Yao, Q. Meng, V. Bulone and Q. Zhou, Flexible and Responsive Chiral Nematic Cellulose Nanocrystal/Poly(ethylene glycol) 
Composite Films with Uniform and Tunable Structural Color, Adv. Mater., 2017, 29(28), 1701323.

147 J. Lv, D. Ding, X. Yang, K. Hou, X. Miao, D. Wang, B. Kou, L. Huang and Z. Tang, Biomimetic Chiral Photonic Crystals, Angew. Chem. Int. Ed., 2019, 58(23), 7783-7787.

148 K. M. Hakansson, A. B. Fall, F. Lundell, S. Yu, C. Krywka, S. V. Roth, G. Santoro, M. Kvick, L. Prahl Wittberg, L. Wagberg and L. D. Soderberg, Hydrodynamic alignment and assembly of nanofibrils resulting in strong cellulose filaments, Nat. Commun., 2014, 5(1), 4018.

149 S. Wang, T. Li, C. J. Chen, W. Q. Kong, S. Z. Zhu, J. Q. Dai, A. J. Diaz, E. Hitz, S. D. Solares, T. Li and L. B. Hu, Transparent, Anisotropic Biofilm with Aligned Bacterial Cellulose Nanofibers, Adv. Funct. Mater., 2018, 28(24), 1707491.

150 H. Lu, A Big Jump in Nacre-inspired Strong, Tough Composites, Chem. Res. Chin. Univ., 2020, 36(3), 488-489.

151 H. Qu, L. L. Yin, Y. S. Ye, X. W. Li, J. W. Liu, Y. Z. Feng, C. Chang, X. P. Zhou, F. C. Tsai and X. L. Xie, Bio-inspired stem-like composites based on highly aligned SiC nanowires, Chem. Eng. J., 2020, 389.

152 Y. Yang, Z. Chen, X. Song, Z. Zhang, J. Zhang, K. K. Shung, Q. Zhou and Y. Chen, Biomimetic Anisotropic Reinforcement Architectures by Electrically Assisted Nanocomposite 3D Printing, Adv. Mater., 2017, 29(11), 1605750.

153 S. Kang, S. Cho, R. Shanker, H. Lee, J. Park, D. S. Um, Y. Lee and H. Ko, Transparent and conductive nanomembranes with orthogonal silver nanowire arrays for skinattachable loudspeakers and microphones, Sci. Adv., 2018, 4(8), eaas8772.

154 Y. Wang, M. Li, Y. Gu, X. Zhang, S. Wang, Q. Li and Z. Zhang, Tuning carbon nanotube assembly for flexible, strong and conductive films, Nanoscale, 2015, 7(7), 3060-3066.

155 W. Xu, Y. Chen, H. Zhan and J. N. Wang, High-Strength Carbon Nanotube Film from Improving Alignment and Densification, Nano Lett., 2016, 16(2), 946-952.

156 Q. Zhu, X. Dong, J. B. Hu, J. S. Yang, X. Y. Zhang, Y. S. Ding and S. M. Dong, High strength aligned SiC nanowire reinforced SiC porous ceramics fabricated by $3 \mathrm{D}$ printing and chemical vapor infiltration, Ceram. Int., 2020, 46(5), 6978-6983.

157 B. Reiser, D. Gerstner, L. Gonzalez-Garcia, J. H. M. Maurer, I. Kanelidis and T. Kraus, Spinning Hierarchical Gold Nanowire Microfibers by Shear Alignment and Intermolecular Self-Assembly, ACS Nano, 2017, 11(5), 4934-4942.

158 W. Wang, X. Sun, W. Wu, H. Peng and Y. Yu, Photoinduced deformation of crosslinked liquid-crystalline polymer film oriented by a highly aligned carbon nanotube sheet, Angew. Chem., Int. Ed., 2012, 51(19), 4644-4647.

159 S. M. Chen, H. L. Gao, Y. B. Zhu, H. B. Yao, L. B. Mao, Q. Y. Song, J. Xia, Z. Pan, Z. He, H. A. Wu and S. H. Yu,
Biomimetic twisted plywood structural materials, Natl. Sci. Rev., 2018, 5(5), 703-714.

160 M. Liu, Y. Pang, B. Zhang, P. De Luna, O. Voznyy, J. Xu, X. Zheng, C. T. Dinh, F. Fan, C. Cao, F. P. de Arquer, T. S. Safaei, A. Mepham, A. Klinkova, E. Kumacheva, T. Filleter, D. Sinton, S. O. Kelley and E. H. Sargent, Enhanced electrocatalytic $\mathrm{CO} 2$ reduction via fieldinduced reagent concentration, Nature, 2016, 537(7620), 382-386.

161 C. Costentin, C. Di Giovanni, M. Giraud, J. M. Saveant and C. Tard, Nanodiffusion in electrocatalytic films, Nat. Mater., 2017, 16(10), 1016-1021.

162 S. Liu, Y. Liu, H. Cebeci, R. G. de Villoria, J. H. Lin, B. L. Wardle and Q. M. Zhang, High Electromechanical Response of Ionic Polymer Actuators with ControlledMorphology Aligned Carbon Nanotube/Nafion Nanocomposite Electrodes, Adv. Funct. Mater., 2010, 20(19), 3266-3271.

163 Z. J. Zhang, Q. Wang, Z. H. Li, Y. C. Jiang, B. Zhao and X. G. Han, Well-aligned BaTiO3 nanofibers via solution blow spinning and their application in lithium composite solid-state electrolyte, Mater. Express, 2019, 9(9), 993-1000.

164 Y. Zhao, J. H. Yan, W. P. Cai, Y. M. Lai, J. Song, J. Y. Yu and B. Ding, Elastic and well-aligned ceramic LLZO nanofiber based electrolytes for solid-state lithium batteries, Energy Storage Mater., 2019, 23, 306-313.

165 M. M. Maitani, C. Xu, K. Hashimoto, Y. Ueda and Y. Wada, Self-oriented $\mathrm{TiO}_{2}$ nanosheets in films for enhancement of electron transport in nanoporous semiconductor networks, Mater. Chem. Front., 2017, 1(10), 2094-2102.

166 L. Huang, Y. He, L. Jin, X. Hou, L. Miao and C. Lü, Fabrication and Properties of Graphene Oxide/Sulfonated Polyethersulfone Layer-by-layer Assembled Polyester Fiber Composite Proton Exchange Membranes, Chem. Res. Chin. Univ., 2018, 34(2), 318-325.

167 W. Liu, S. W. Lee, D. C. Lin, F. F. Shi, S. Wang, A. D. Sendek and Y. Cui, Enhancing ionic conductivity in composite polymer electrolytes with well-aligned ceramic nanowires, Nat. Energy, 2017, 2(5), 17035.

168 J. L. Wang, Y. R. Lu, H. H. Li, J. W. Liu and S. H. Yu, Large Area Co-Assembly of Nanowires for Flexible Transparent Smart Windows, J. Am. Chem. Soc., 2017, 139(29), 9921-9926.

169 B. J. Liu, J. Zheng, J. L. Wang, J. Xu, H. H. Li and S. H. Yu, Ultrathin $\mathrm{W} 18 \mathrm{O} 49$ nanowire assemblies for electrochromic devices, Nano Lett., 2013, 13(8), 3589-3593.

170 Q. X. Chen, Y. H. Liu, X. Z. Qi, J. W. Liu, H. J. Jiang, J. L. Wang, Z. He, X. F. Ren, Z. H. Hou and S. H. Yu, Ordered Nanostructure Enhances Electrocatalytic Performance by Directional Micro-Electric Field, J. Am. Chem. Soc., 2019, 141(27), 10729-10735. 